\title{
INFINITO ACTUAL E INCONSISTENCIAS: ACERCA DE LAS INCOHERENCIAS EN LOS ESQUEMAS CONCEPTUALES DE ALUMNOS DE 16-17 AÑNOS
}

\author{
GARBIN, SABRINA y AZCÁRATE, CARMEN \\ Departament de Didàctica de les Matemàtiques i les Ciències Experimentals \\ Universitat Autònoma de Barcelona \\ sgarbin@usb.ve \\ carmen.azcarate@uab.es
}

\begin{abstract}
Resumen. En este artículo presentamos algunos resultados, reflexiones y aportaciones de un trabajo de investigación (Garbin, 2000) que se centra en identificar las inconsistencias y representar, categorizar y analizar las situaciones de coherencia que manifiestan los alumnos en relación con sus esquemas conceptuales asociados al concepto de infinito actual, el cual se contextualizan en problemas expresados en lenguajes matemáticos diferentes: verbal, geométrico, gráfico, algebraico y analítico. Metodológicamente la investigación se enmarca en un estudio cualitativo. El análisis de datos es inductivo y el foco de investigación tiene un carácter exploratorio, descriptivo e interpretativo. Participaron en el estudio 80 estudiantes de 16-17 años.

Palabras clave. Infinito, esquemas conceptuales, inconsistencias, incoherencias.
\end{abstract}

Summary. We present in this article some of the results, considerations and contributions contained in a research work (Garbin, 2000) focused on the identification of inconsistencies and on representation, categorization and analysis of situations of coherence showed by students, in relation to their concept schemes associated to the concept of present infinity, within the context of problems set forth through different mathematical languages: verbal, geometrical, graphical, algebraic and analytical. From a methodological point of view this research is framed within a qualitative study. The data analysis is inductive and its aim is exploratory, descriptive and interpretative. Eighty 16-17 year old students took part in this study.

Keywords. Infinity, concept images, inconsistency, incoherency.

\section{INTRODUCCIÓN}

La preocupación por las dificultades del aprendizaje y de la enseñanza de los conceptos matemáticos en el bachillerato, en los cursos preuniversitarios y en los primeros de la universidad, nos hizo escoger una investigación que, desde el punto de vista de las concepciones de los alumnos y alumnas, indaga y examina los motivos que hacen de la problemática de un concepto matemático, un tema para explicar y comprender.

Como problemática, nos ha interesado el concepto de infinito matemático. Podemos hacernos la siguiente pregunta: ¿Por qué elegir este concepto? Podríamos dar dos razones: la importancia que tiene el concepto tanto en sí mismo como en la enseñanza de la matemática. Sobre esta última queremos recordar una frase de Dalessert, citada en D'Amore (1996): «La enseñanza de la matemática debe dirigirse hacia dos objetivos que le son propios: el sentido del rigor lógico y la noción de infinito». De manera especial, a nosotras nos ha interesado el trinomio: infinito - lenguajes matemáticos inconsistencias.

Al principio del bienio 1996-98, nos planteamos como problema de estudio el interrogante de cuál es la posible influencia de los lenguajes matemáticos en la concepción del infinito actual y en las inconsistencias de los alumnos. Las preguntas que nos hacíamos entonces eran las siguientes: ¿Cómo se describen estas inconsisten- 
cias? ¿Pueden ser categorizadas? ¿Qué tipo de conexiones se pueden establecer entre lo verbal, geométrico, gráfico, algebraico y numérico en el concepto de infinito actual? Con estas preguntas en el horizonte, realizamos un estudio previo exploratorio (Garbin, 1998; Garbin y Azcárate, 2000), en que pudimos constatar la influencia de los lenguajes matemáticos implicados en los problemas aplicados a los estudiantes, tanto en la concepción del infinito actual como en las inconsistencias asociadas. Los resultados nos han sugerido también que la intuición del infinito actual es «sensible» no sólo al contexto (Fischbein, Tirosh y Hess, 1979), sino también a las imágenes conceptuales asociadas a otros conceptos implicados en las cuestiones, en este caso el de función y suma infinita. También mostramos que en las preguntas en que está implicado un proceso de infinitud, los estudiantes no siempre responden teniendo en cuenta el proceso infinito o evadiendo la infinitud, respondiendo de manera finita. A las categorías de respuestas detectadas anteriormente a partir de problemas de divisibilidad infinita (Fischbein, Tirosh y Hess, 1979; Núñez, 1994: el proceso termina; el proceso es infinito; el proceso termina pero en la teoría no; y los que no contestan), nosotras agregamos una categoría formada por las respuestas de un grupo de alumnos que, para fundamentarlas, no consideran el proceso de división infinito implicado en la cuestión: «el proceso de división finito o infinito no determina la respuesta del alumno».

Esta experiencia nos abrió nuevos interrogantes y matizó el planteamiento de la investigación, que pretendía identificar las inconsistencias y representar, categorizar y analizar las situaciones de coherencia que manifiestan los alumnos en relación con sus esquemas conceptuales asociados al concepto de infinito actual y que se contextualizan en problemas expresados en lenguajes matemáticos diferentes: verbal, geométrico, gráfico, algebraico y analítico.

En este artículo presentamos algunos de los resultados, aportaciones y reflexiones de esta última investigación (Garbin, 2000), principalmente enfocados a dejar en evidencia la importancia de describir y distinguir los términos inconsistencia e incoherencia, describir lo que entendemos por tarea de conexión y reflexionar sobre su posible importancia en la actividad matemática, en la búsqueda de cómo ayudar al desarrollo de un pensamiento coherente en el estudiante $\mathrm{y}$, en consecuencia posteriormente, tal vez de un pensamiento consistente respecto al concepto que se esté trabajando, en nuestro caso, el infinito actual.

\section{FUNDAMENTACIÓN TEÓRICA Y ANTECE- DENTES}

El tema de estudio, la naturaleza del problema, el propósito de la investigación y los objetivos planteados son, entre otros, los que ofrecieron la perspectiva teórica del trabajo.
Por motivos obvios de extensión no podemos presentar en su totalidad el trabajo teórico, pero sí podemos presentar la fundamentación y enmarque teórico que están centrados en la teoría cognitiva desarrollada por Tall y Dreyfus con relación al desarrollo y crecimiento del pensamiento matemático avanzado. Decidimos enfatizar aquellos aspectos que hemos considerado importantes para fundamentar nuestra investigación. Los conceptos tratados fueron, de manera particular, el concept image y el concept definition (Tall y Vinner, 1981), los cuales traducimos como esquema conceptual y definición del concepto (Azcárate, 1990); también el concepto de procepto (Gray y Tall, 1994) en torno a la dualidad del proceso y concepto. En cuanto a procesos podemos distinguir entre: a) los implicados en la representación como son el proceso propiamente dicho, las representaciones y translaciones y la modelización; y $b$ ) los implicados en la abstracción, es decir, generalizar, sintetizar, abstraer. También nos hemos detenido a describir los procesos del crecimiento cognitivo en la etapa de transición desde la matemática elemental hacia la avanzada, considerando que «el lugar donde el pensamiento matemático elemental se convierte en avanzado no se ha definido todavía con precisión» (Tall, 1995).

Esta teoría era necesaria, pero no suficiente, para abordar temas como la intuición, las inconsistencias y las representaciones, de especial importancia en nuestro trabajo. Se hizo, pues, necesario completar el marco con una mirada más profunda hacia estos términos que acabamos de mencionar.

La importancia que ha tenido y tiene la noción de intuición en la matemática, en las ciencias y en la educación matemática, hizo que surgiera un gran interés por investigarlo, especialmente considerando el conocimiento intuitivo como un camino básico, junto al analítico, en la actividad matemática, como mostró Fischbein en su artículo «Intuition and Proof» (1982). Esta importancia podría ser la respuesta obvia a por qué escoger esta noción en el marco teórico, además de considerar que en las preguntas que hemos planteado a nuestros alumnos «entra en juego» la intuición. Pero, alguna razón más hemos encontrado al centrarnos en la noción de infinito.

Sabemos que el concepto aristotélico de infinito es una noción potencial que dominó en la historia hasta la época cantoriana, y que tuvo una gran influencia en el desarrollo de este concepto. Como ha sido expresado por Fischbein (1982) este concepto potencial de infinito es el que responde a la interpretación natural intuitiva del infinito. «Un objeto potencialmente infinito (por ejemplo, una línea que puede ser extendida indefinidamente) tiene un significado «conductual». Una operación potencialmente infinita también tiene un significado «conductual» (por ejemplo, dividir indefinidamente un segmento de línea). Un infinito actual no tiene un significado conductual, por tanto, no es congruente con una interpretación intuitiva.» (p. 13).

Debemos advertir que las respuestas correctas a los problemas de nuestro cuestionario resultan ser contrain- 
tuitivas; por otra parte, «las intuiciones no son absolutas, dependen del contexto...» (Fischbein, 1998, p. 367). Dado el interés de nuestra investigación y lo expresado anteriormente, resultó imprescindible considerar la intuición, su rol, su clasificación y sus características. Fischbein (1998) describe las siguientes características generales de las cogniciones intuitivas: $a$ ) cogniciones directas, autoevidentes; $b$ ) certeza intrínseca; $c$ ) coercisión; d) capacidad de extrapolar; e) globalidad. También clasifica a las intuiciones como de aceptación y anticipación, las primeras de las cuales pueden ser primarias y secundarias.

El tema de inconsistencias tiene una relevancia especial en nuestro estudio. En el año 1990, varios investigadores de didáctica de la matemática dedicaron un lugar especial al tema de las inconsistencias en un volumen monográfico de la revista Focus on Learning Problems in Mathematics. El objetivo principal y el deseo de los investigadores era que dicho volumen sirviera para enriquecer el conocimiento básico sobre el tema y animar a seguir investigando en este dominio. Los intereses comunes a todos los artículos son los posibles orígenes de las inconsistencias y el sugerir estrategias instruccionales para promover el aprendizaje a través de ellas. Algunos presentaban investigaciones empíricas (Wilson, Tall, Tirosh y Graeber) mientras otros ofrecían discusiones teóricas (Behr y Harel; Vinner). Steffe, por otra parte, trata de este tema desde un punto de vista constructivista y Tirosh provee una sinopsis sobre el rol de las inconsistencias en el aprendizaje de la matemática.

Hemos querido dar a conocer aquellas aportaciones y conclusiones que consideramos más importantes para nuestro trabajo y que provienen de las investigaciones antes mencionadas. Subrayamos de manera especial la clasificación que hace Tirosh de las ideas inconsistentes de los estudiantes, ya que permite situar las observadas en nuestra investigación.

Vinner (1990) había distinguido dos tipos de inconsistencias: las que se derivan de un sistema formal y las que suceden desde un punto de vista psicomatemático. Consideró dos niveles de inconsistencias: las superficiales y las profundas. Las primeras, derivadas de un error en una demostración o como resultado de un axioma contradictorio con otro; y las profundas, que pueden ser derivadas de un concepto mal formulado o debidas a la presencia de conexiones implícitas erróneas. Tirosh aumenta esta clasificación ofreciendo otros tipos de ideas inconsistentes, de particular importancia para la instrucción: a) inconsistencias directas e indirectas; $b$ ) validez matemática de las proposiciones; $c$ ) inconsistencias externas e internas; d) consciencia de los estudiantes de sus inconsistencias.

En cuanto a la falta de consciencia de los estudiantes sobre sus ideas inconsistentes, es una situación identificada y que preocupa en cuanto no ayuda a que los alumnos puedan aprovechar sus inconsistencias para su aprendizaje y hace que el método de crear un conflicto cognitivo al estudiante no siempre tenga los resultados deseados.
Con relación a los posibles orígenes de inconsistencias de las ideas matemáticas de los estudiantes, Tirosh sigue las misma áreas consideradas por Tall, ofreciendo una subclasificación que podemos ver en la tabla siguiente:

\begin{tabular}{|c|}
\hline La matemática: naturaleza relativa \\
\hline $\begin{array}{l}\text { La mente: conocimiento y creencias } \\
\text { - Discrepancia entre el aprendizaje formal, intuitivo y el conoci- } \\
\text { miento algorítmico. } \\
\text { - Discrepancia entre el esquema conceptual y la definición del } \\
\text { concepto. } \\
\text { - Naturaleza del contexto del conocimiento adquirido. } \\
\text { - Resistencia al cambio conceptual. } \\
\text { - Percepción del estudiante de la matemática. }\end{array}$ \\
\hline $\begin{array}{l}\text { El mensaje: el lenguaje, el currículo y la instrucción. } \\
* \text { No son mutuamente excluyentes. }\end{array}$ \\
\hline
\end{tabular}

En nuestra investigación también son importantes los términos lenguajes matemáticos, contexto y representación. De ahí que hayamos abordado el estudio del término representación. Janvier, en el año 1987, editó el libro Problems of representation in the teaching and learning of mathematics, que destacó en ese tiempo el tema de las representaciones en la educación matemática y situó la perspectiva teórica del tema en ese momento.

Para complementar nuestro marco teórico hubiésemos podido centrarnos en la perspectiva teórica que ofrecen los artículos citados anteriormente. Sin embargo, sin quitarle importancia a tales aportaciones y sin olvidar la resonancia de estos planteamientos, nos hemos centrado en la teoría de las representaciones semióticas, desde el punto de vista cognitivo, desarrollada por Duval (1993, 1996, 1999a, 1999b), que llevó a afirmar que «la característica esencial de la actividad matemática es el cambio de registro de representación y que la conversión de las representaciones es un problema crucial en el aprendizaje de las matemáticas» (Duval, 1999a, 1999b). Hemos presentado aquellos términos y definiciones que son básicos para entender las aplicaciones subsiguientes al analizar la actividad matemática desde el punto de vista de las representaciones semióticas. Específicamente para nuestro trabajo, los términos que más nos interesan clarificar son: representaciones semióticas, registro de representación, conversión y coordinación de las representaciones.

Antes de centrarnos en la presentación de algunas de las investigaciones de interés didáctico realizadas sobre el infinito matemático, dimos un paseo por la historia del concepto de infinito con especial interés por el infinito actual. Este paseo lo hemos dividido en tres partes. En la primera hemos contextualizado las dos épocas históricas que enmarcaron de manera especial el trabajo y el desarrollo de tal concepción en las grandes mentes matemáticas. La segunda parte la centramos en la noción de infinito matemático con una mirada especial al infinito actual y la tercera la hemos dedicado al matemático 
Zenón de Elea. También presentamos dos recientes aportaciones acerca de las paradojas de Zenón (Harrison, 1996; Alper y Bridger, 1997). Para este paseo seguimos de cerca la exposición de Boyer (1992), así como el trabajo epistemológico y las reflexiones realizadas por Waldegg en su tesis doctoral (1987). También tomamos en cuenta la exposición que hace Rucker (1995) en el primer capítulo de su libro Infinity and the Mind y la de Moreno y Waldegg (1991), entre otros.

Son muchas las investigaciones realizadas en didáctica sobre el tema del infinito matemático. Tall (1992), al referirse a la transición del pensamiento matemático elemental al pensamiento matemático avanzado, en cuanto a funciones límites, demostración e infinito, presentó una serie de investigaciones realizadas sobre ese tema. Tall cita la existencia de investigaciones basadas en las inconsistencias que existen entre el infinito cardinal de Cantor y las intuiciones. Tales investigaciones tratan de las concepciones y conflictos presentes en la mente de los estudiantes; nombra explícitamente las siguientes: Tall (1980); Fischbein, Tirosh y Hess (1979); Tirosh (1991) y Sierspinska (1987). Más tarde, D’Amore (1996) recogió un centenar de investigaciones que se han venido desarrollando en los últimos años, completando la lista presentada por Tall pero con un objetivo distinto: mostrar algunas direcciones que ha tomado la investigación en la didáctica del infinito matemático, a través de una exposición que no toma como referencia los distintos conceptos del infinito y las concepciones correspondientes, como lo hizo Tall, sino la referencia histórica del desarrollo del concepto. En este artículo podemos encontrar investigaciones sobre las paradojas de Zenón y sobre algunas consideraciones de Galileo; investigaciones aristotélicas y sobre los usos «actual»y «potencial» del infinito; investigaciones sobre el continuo, alrededor de la adquisición del infinito como medida y conteo, sobre algunas sumas infinitas de Oresme y Bolzano; otras que tratan la inducción; y, por último, investigaciones realizadas en el marco de la psicología del aprendizaje. El año siguiente (D'Amore, 1997) decidió publicar la bibliografía que posee sobre el tema, completando la presentada el año anterior. Consciente de que es un tema muy estudiado, la llama bibliografía in progress y presenta en ella 280 títulos que representan trabajos o investigaciones de interés histórico, epistemológico, psicológico, filosófico o didáctico, realizados a partir del año 1951 hasta la fecha de publicación del artículo. Algunos de los artículos están orientados hacia el concepto mismo, y muchos otros, hacia la comprensión de otros conceptos matemáticos, en los cuales el infinito está implícito en los procesos cognitivos de aprendizaje de los mismos. Podemos añadir nuevos artículos publicados entre los años 1996-97 a esta bibliografía de trabajos vinculados al concepto de infinito: Riscos (1996); Stavy y Tirosh (1996); Blazek y Sitia (1997), de orientación matemática; Mura y Maurice (1997); Harrison (1996) y Alper y Bridger (1997), de orientación filosófico-matemática.

Algunas de las investigaciones son consideradas piloto y pilares en el área de la didáctica del infinito matemático, como lo son Fischbein, Tirosh y Hess (1979), Tall
(1980), Tirosh (1991) y Moreno y Waldegg (1991). Otras dos más recientes, la primera, con una aportación desde un punto de vista psicológico (Nuñez, 1994), y la segunda, con un enfoque sobre metacognición y consistencia en el caso del infinito (Tsamir y Tirosh, 1995), han sido de particular interés para nosotras.

Con la presentación bibliográfica hemos querido resaltar la gran cantidad de literatura existente sobre el infinito matemático. Así, tenemos una idea sobre la gran variedad de temática e intereses surgidos a través del tiempo. Hemos podido constatar que las investigaciones dedicadas específicamente a la intuición del infinito matemático, desde un interés didáctico y psicológico, hacen importantes aportaciones al conocimiento de las concepciones del infinito que presentan los alumnos. Además, ponen de manifiesto lo intuitivamente contradictorio que resulta ser este concepto y evidencian la presencia de inconsistencias en las respuestas de los alumnos, básicamente influenciadas por el contexto, en problemas en que está presente esta noción.

No nos queda duda de la importancia, para la didáctica de la matemática, de conocer la interacción entre lenguajes matemáticos - representación - inconsistencias infinito, pero también es cierto que aún queda mucho por explorar en este campo específico. Con este entorno, queremos nuevamente subrayar la importancia y la novedad de nuestro trabajo, que seguimos, en parte, presentando en este artículo.

\section{METODOLOGÍA}

La investigación se enmarca en un estudio de tipo cualitativo. El análisis de datos es inductivo, ya que las categorías e interpretaciones se construyeron a partir de la información obtenida. El foco de investigación tiene un carácter exploratorio, descriptivo e interpretativo. Estas características son parte -y son propias- de la «investigación cualitativa» (Latorre et al., 1996).

Antes de esta investigación se había llevado a cabo una experiencia piloto que llamamos investigación previa (Garbin, 1998; Garbin y Azcárate, 2000). De este estudio exploratorio revisamos la metodología, los instrumentos de recogida de datos y análisis de éstos, y se corrigieron y completaron aquellos aspectos que así lo requerían. Realizamos la investigación con nuevos y con mayor número de participantes, y se construyeron nuevas categorías.

\section{Instrumentos de recogida de datos y descripción de los participantes}

La investigación se realizó con 80 estudiantes de $2^{\circ}$ de bachillerato de tres centros educativos (dos grupos de 27 alumnos y uno de 26), cuyas edades estaban comprendidas entre 16 y 17 años. En el momento de la aplicación de los cuestionarios, los estudiantes no tenían conocimientos formales previos sobre límites; hasta ese mo- 
1.- Observa la siguiente figura.

Nos muestra un esquema en el que se biseca cada vez el segmento de la derecha, es decir los puntos $\mathrm{M}, \mathrm{N}, \mathrm{O}, \mathrm{P}$, son los puntos medios de los segmentos $\mathrm{AB}, \mathrm{MB}, \mathrm{NB}$ y OB respectivamente.

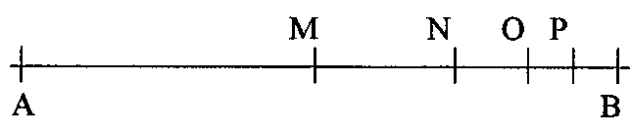

Si se siguen haciendo más y más bisecciones.

¿Crees que es posible llegar a una situación en la que un punto de la bisección coincide con el punto B?

Explica tu respuesta.

2.- Se deja caer una pelota desde 2 metros de altura sobre una superficie horizontal. Cada vez que la pelota llega al suelo, tras caer desde una altura $h$, rebota hasta una altura $\mathrm{h} / 2$.

¿Podrías calcular la distancia total recorrida por la pelota?. Explica tu respuesta ¿Podrías decir cuantos rebotes hará la pelota?. Explica tu respuesta.

3.- Considera la siguiente suma

$$
1+1 / 2+1 / 4+1 / 8+1 / 16+\ldots+\ldots
$$

¿Cuál crees que es el valor de esta suma?. Explica tu respuesta.

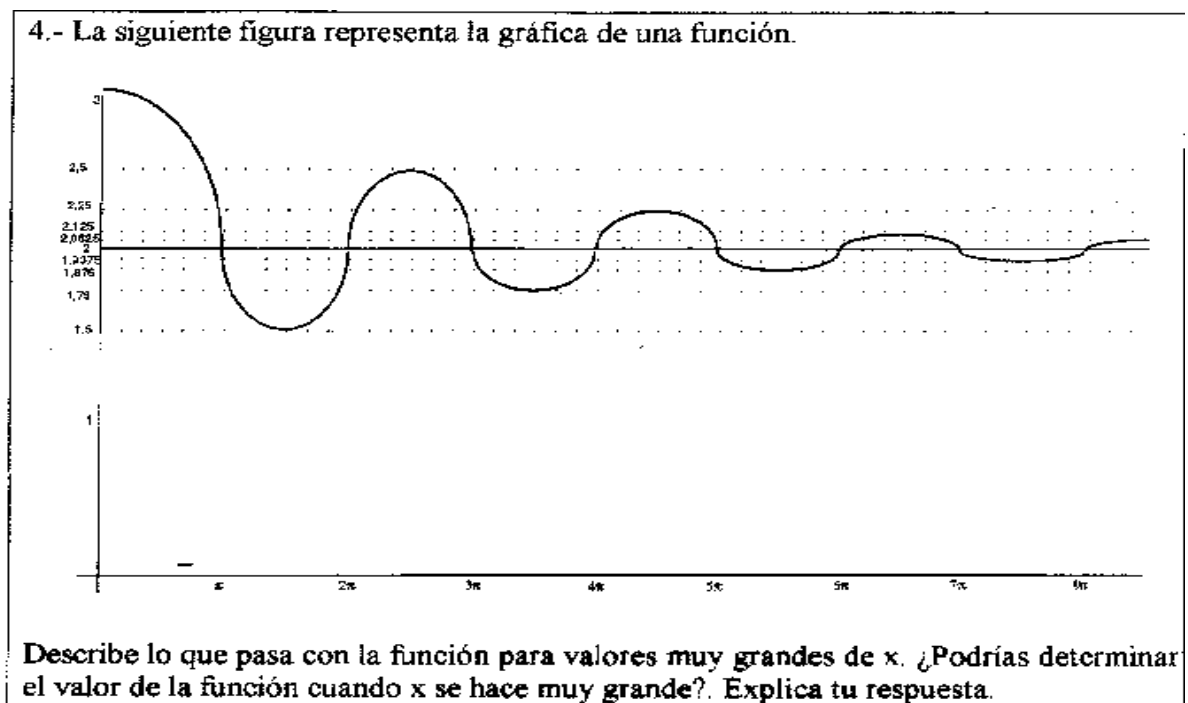

el valor de la función cuando $x$ se hace muy grande? Explica tu respuesta.

5.- Considera la siguiente ecuacion

$$
y=1+1 / 2+1 / 2^{2}+1 / 2^{3}+1 / 2^{4}+\ldots+1 / 2^{n}+\ldots
$$

¿Podrias decir para qué valor de $n$ resulta $y=2$ ? Explica tu respuesta 
mento, las unidades didácticas desarrolladas en clase por los profesores habían sido: el número real (números reales y errores); trigonometría (razones trigonométricas, resolución de triángulos y ecuaciones trigonométricas); estudio descriptivo de las funciones elementales (polinomios, funciones polinómicas, racionales y de proporcionalidad inversa); función real.

Dos cuestionarios fueron aplicados a los estudiantes: $\mathrm{C}_{1}$ $\mathrm{y} \mathrm{C}_{2}$.

El primer cuestionario, $\mathrm{C}_{1}$ (Fig. 1), consta de 5 preguntas en las que está presente el mismo concepto matemático, el infinito actual, y los problemas son de divisibilidad infinita. La particularidad de la mayoría de las preguntas consiste en ser una versión actualizada, diferenciada por el contexto, de la primera paradoja de Zenón de la división. Otras generan la misma paradoja. Cinco lenguajes matemáticos distintos diferencian el contexto de cada una: geométrico, verbal, analítico, gráfico y algebraico. Cada problema, en su enunciado, tiene una parte que usa la lengua natural como registro lingüístico, al explicar el problema y la cuestión planteada y otra parte que usa otro registro de representación semiótica. Como se puede observar, los registros de representación semiótica (indicamos RRL para indicar registro de representación lingüístico y RR registro de representación no lingüístico) que aparecen en el enunciado son:

a) RR: figura geométrica (segmento de recta);

b) RRL: lengua natural;

c) RRL: numérico (suma infinita);

d) RR: gráfico cartesiano (función cartesiana);

e) RRL: escritura algebraica (ecuación con suma infinita).

El segundo cuestionario, $\mathrm{C}_{2}$ (Fig. 2), se aplicó una semana después de pasar el $\mathrm{C}_{1}$. El objetivo de este cuestionario era permitir a los estudiantes que tomaran posición ante sus propias respuestas, darles la oportunidad de corregir, matizar y ampliar las respuestas dadas en $C_{1}$ y relacionar o encontrar similitud entre las preguntas o respuestas. Por otra parte, considerar que podían comunicarse entre ellos, reflexionar sobre lo ocurrido en el cuestionario y poder corregir o completar nuevamente las respuestas días después, pensábamos que podía ser beneficioso para evitar aquellas respuestas ingenuas o producto de una «conducta pseudoanalítica» (Vinner, 1997). En este tipo de problemas los registros de representación tienen un fuerte carácter de estímulo visual, que puede inducir al alumno a tener este tipo de conducta.

El cuestionario se aplicó de la siguiente manera:

-Se entregó a cada estudiante su propio cuestionario $\left(C_{1}\right)$ contestado días antes, un bolígrafo rojo y el nuevo cuestionario $\left(\mathrm{C}_{2}\right)$.

-Se explicó a los alumnos el objetivo de esta nueva aplicación, la oportunidad que tenían para pensar nuevamente sobre la tarea realizada días antes y corregir o matizar sus respuestas en caso de creerlo conveniente; además de este objetivo, el interés de recoger nueva información a partir de la segunda pregunta del nuevo cuestionario.

- El cuestionario $\mathrm{C}_{2}$ consta de dos parte (a) y (b). Se les pidió contestar la parte (a) en $\mathrm{C}_{1} \mathrm{y}$ la parte (b) en $\mathrm{C}_{2}$.

- El uso del bolígrafo rojo se justificó explicando que permitía no confundir las respuestas dadas en un primer momento y las correcciones realizadas por los alumnos sobre las propias respuestas.

Esta experiencia la hemos considerado positiva, ya que permitió la ubicación definitiva de todos los estudiantes en las líneas de coherencia (ver apartado, un instrumento: las líneas de coherencia) y por la conducta observada en los estudiantes durante esta nueva situación. Lejos de causar rechazo el «pensar sobre lo mismo», los alumnos se mostraron más atentos y más concentrados en la nueva tarea. Hemos interpretado que el asombro que les causó la posibilidad de revisar las propias respuestas y reflexionar de nuevo acerca de las preguntas fue lo que favoreció esta conducta, distinta (cualitativamente) a la que habíamos observado en la aplicación del primer cuestionario.

Un tercer instrumento de recogida de datos ha sido el de las entrevistas grabadas. Es un tipo de entrevista semiestructurada y dirigida. La entrevistadora acudió a la entrevista con un guión, que fue el mismo para todos los alumnos. Las entrevistas tuvieron una duración comprendida entre 30 y 45 minutos. Los problemas escogidos para la entrevista han sido tres del $\mathrm{C}_{1}$ (preguntas 1 , 3 y 4), y un cuarto problema, también de este cuestionario (pregunta 2), que se propone al estudiante después de la resolución de los tres anteriores.

Dado que se necesita el conocimiento de las categorías establecidas durante el estudio y del trabajo realizado, explicaremos más adelante los criterios seguidos para la selección de los 6 estudiantes entrevistados y en los anexos mostraremos el guión. Se hizo un análisis descriptivo e interpretativo de cada entrevista.

Figura 2

Instrumento de recogida de datos. Cuestionario.

Segundo cuestionario

Tienes el cuestionario que tu mismo/a has contestado: a) Usando un bolígrafo rojo puedes ahora corregir o completar tus respuestas.

Si corriges o completas, explica por qué lo haces y, en caso que no lo hagas, explica también el motivo.

(Si no habías contestado alguna de las prguntas inténtalo ahora.)

La respuesta que das a cada pregunta, ¿crees que es la única respuesta posible?

b) ¿Encuentras que existe alguna relación o conexión entre las preguntas? ¿Y entre las respuestas? Explícalo. 


\section{Un instrumento: las líneas de coherencia}

Optamos por el uso de las redes sistémicas (Bliss y Ogborn, 1979; Bliss, Monk y Ogborn, 1983; Azcárate, 1990; Romero, 1996; Garbin, 1998) como sistema de representación de los datos cualitativos obtenidos a partir de las respuestas dadas por los alumnos en $\mathrm{C}_{1}$. Las redes sistémicas posibilitan una configuración de los datos que permite mirar todas las respuestas efectivas de los alumnos encuestados. Estos datos nos interesaban para acercarnos a los esquemas conceptuales de los estudiantes asociados al concepto de infinito actual.

Se estableció una red sistémica por cada pregunta del cuestionario y se hizo un análisis descriptivo e interpretativo pregunta por pregunta. Los alumnos habían sido identificados aleatoriamente con números del 1 al 80.

Construimos unas tablas resumen a partir de las redes sistémicas diseñadas con las respuestas obtenidas en $C_{1}$. Éstas se construyeron, considerando prácticamente la misma organización establecida en las redes sistémicas, por categorías independientes. Se siguió la metodología empleada en el estudio previo (Garbin, 1998; Garbin y Azcárate, 2000).

A partir de las respuestas de los alumnos, representadas en las tablas-resumen, y del contexto de las mismas, expresado en los lenguajes matemáticos distintos (geométrico, verbal, analítico, gráfico y algebraico), construimos un instrumento que permite mostrar las respuestas coherentes o incoherentes de los estudiantes.

Para la construcción hemos tomado en cuenta los procesos explicitados en Núñez (1994). En el concepto involucrado en las cinco preguntas, están presentes dos tipos de iteracciones, la divergente y la convergente, es decir, que el número de divisiones crece mientras la distancia disminuye. Pero, por otro lado, está la naturaleza del contenido que tiene dos tipos de atributos: el número de divisiones referido a cardinalidad y la distancia cubierta referida a espacio. Por ejemplo, en la pregunta 1, el proceso de bisección es divergente y el número de divisiones que se hacen para que el punto de bisección alcance al punto extremo B se refiere a la cardinalidad. Por otro lado, la distancia que cubre cada división disminuye, debido a que cada vez se van haciendo segmentos más pequeños; ésta sería la iteración convergente.

A partir de las respuestas hemos establecido tres líneas de coherencia que hemos llamado: línea finitista (o de evasión de la infinitud), línea actual y línea potencial (Figs. 6, 7, 8 y 9, en el anexo). Como podemos observar, estos gráficos están formados por tablas entrelazadas entre sí, las cuales han sido diseñadas a partir de las tablas resumen. Si se sigue el orden dado por estos enlaces, se pueden identificar a aquellos alumnos que no mantienen respuestas coherentes entre las cinco preguntas del cuestionario.

Observando cada línea podemos conocer las respuestas dadas por los alumnos, las cuales pueden ser correctas o no, consistentes o no con el concepto involucrado en los problemas. Mencionaremos aquellos alumnos y sus respuestas que no aparecen en las líneas anteriormente expuestas:

1) En la pregunta 1, contesta tanto afirmativamente como negativamente. Ambas respuestas en sentido potencial y actual se han considerado válidas. Estos alumnos deberían contestar de forma paradójica en todas las preguntas.

2) En la pregunta 1, distingue entre la teoría y la prácti$c a$, en el sentido de la primera paradoja de Zenón, que es inducida por el tipo de infinito involucrado en $\mathrm{C}_{1}$. Estos alumnos deberían distinguir entre la teoría y la práctica en todas las preguntas.

3) En la pregunta 1, distingue entre la representación geométrica y numérica, en el mismo sentido de la respuesta (b), pero al justificar distingue una identificación numérica y geométrica en el registro de representación usado en la pregunta. Estos alumnos deberían distinguir las respuestas dependiendo de la identificación que se pueda hacer de la representación de cada pregunta.

4) No contesta. Esta clase aparece en muchas de las tablas construidas. Estos alumnos pueden tener cualquier tipo de respuestas en las preguntas que contesten.

5) En la primera pregunta responde que el punto de bisección no alcanza al punto B por no poder ser éste un punto de bisección, porque es punto extremo del segmento. Esta respuesta no ha sido determinada por la infinitud, sino por un obstáculo cognitivo. En las otras respuestas, el alumno podría seguir en cualquiera de las tres líneas establecidas.

\section{RESULTADOS}

\section{Categorización de los estudiantes según las líneas de coherencia}

Las líneas de coherencia permiten clasificar la situación de coherencia o incoherencia de cada estudiante. Las respuestas coherentes, en el sentido de cada línea, son las que han determinado la clasificación:

a) Si un alumno tiene tres o más respuestas coherentes según una línea determinada, se le sitúa en la categoría de la línea correspondiente.

b) $\mathrm{Si}$ un estudiante tiene tres respuestas coherentes en una de las líneas y tres en una línea distinta, se le coloca en la categoría compartida por ambas líneas.

c) Si un alumno tiene sus respuestas situadas en diferentes líneas, se le sitúa en una categoría que hemos llamado mixta.

A partir del análisis descriptivo e interpretativo hemos encontrado lo siguiente: 
- Los alumnos finitistas o que evaden la infinitud (categoría 1):

a) En preguntas de tipo geométrico, algebraico o numérico, difícilmente contestan de forma infinitista en preguntas en que la infinitud se presenta en situaciones de la vida real y que la coherencia de pensamiento es inducida por espacios acotados o expresiones no acotadas.

b) Tienen respuestas a la pregunta $1\left(\mathrm{C}_{1}\right)$ del tipo $\mathrm{B}, \mathrm{T}$ y $\mathrm{P}, \mathrm{G}$ y $\mathrm{N}$ o $\mathrm{S}$ y $\mathrm{N}$. El conflicto entre finito e infinito tiene un grado de intensidad superior al grupo anterior y la concepción del infinito es básicamente potencial.

c) La noción de infinito actual aparece a partir de representaciones que inducen a ello (como, por ejemplo, espacios acotados, analogía de conceptos finitos, considerar que la suma infinita debe tener un valor numérico finito).

Con estos criterios hemos obtenido las 5 categorías de alumnos:

1) alumnos que están situados en la categoría 1;

2) alumnos que están situados en la categoría 2 ;

3) alumnos que están situados en la categoría 3;

4) alumnos que están situados en la categoría 1/3;

5) alumnos que están situados en la categoría mixta.

Estas categorías se pueden visualizar en las figuras 10 a 14 del anexo.

Para visualizar el recorrido de cada alumno según las líneas de coherencia, hemos utilizado una línea continua y la simbología que aparece en la figura 15 del anexo para representarlo. La representación de la línea 1 es distinta a la de las líneas 2 y 3 , debido a que la línea 1 se subdivide en 1.1 y 1.2 , determinadas por las respuestas a la pregunta 2, partes (a) y (b).

- En la mayoría de las respuestas de los alumnos actualistas (categoría 2), la concepción actual entra en conflicto sobre todo en la pregunta $2\left(\mathrm{C}_{1}\right)$, contexto «realfísico».

- Los alumnos potencialistas en la mayoría de sus respuestas (categoría 3) responden básicamente con la intuición natural del infinito.

Del resto de categorías hablaremos más adelante.

\section{Inconsistencias e incoherencias}

Cuando se habla de una idea o pensamiento inconsistente es con relación al concepto matemático involucrado, o a contradicciones dentro de una teoría matemática dada. Generalmente aparecen durante la resolución de un problema o en una respuesta al mismo.

Una forma particular de inconsistencias directas, según la clasificación de Tirosh (1990), son las que se presentan en la situación en que los estudiantes tienen que resolver un mismo problema representado de diferente forma, poniendo de manifiesto que los alumnos no mantienen respuestas consistentes ante varias representaciones. Es decir, alguna respuesta puede ser consistente con el concepto para un tipo de representación e inconsistente con el concepto para una representación distinta.

En esta situación particular en que el estudiante tiene que resolver un mismo problema representado de diferentes formas, como ha sido el caso de nuestro cuestionario, nosotros introducimos el término de incoherencia, pero con un matiz distinto al de inconsistencia que hace que podamos usar de manera diferente ambos términos aunque estén estrechamente relacionados. Trataremos de explicar lo anterior a continuación.

Tirosh (1990), al hablar de situaciones como las mencionadas anteriormente, describe el siguiente ejemplo: en un estudio, los alumnos argumentaron que $x=3$ es una función y al mismo tiempo no lo es, dado que la función estaba representada en un gráfico cartesiano y también estaba descrita en un diagrama de flechas. En este ejemplo estamos hablando de dos tipos de gráficos como registros de representación. Se presenta una situación un poco distinta cuando estos gráficos aparecen en dos problemas donde, tal vez, uno tenga un enunciado en lenguaje natural y el segundo, en un lenguaje formal. La contextualización del problema, entonces, influye en su comprensión.

En matemáticas se habilitan diferentes lenguajes matemáticos, como lo son el algebraico, analítico, geométrico, gráfico y verbal; éstos son los que hemos escogido para nuestros problemas. Cada uno de estos lenguajes genera contextos lingüísticos matemáticos diferentes. Y cada lenguaje matemático utiliza una combinación de ciertos registros de representación semiótica que pueden ser del tipo lingüístico (lenguaje natural, escritura algebraica, lenguaje formal...) o de otro tipo (figuras geométricas, gráficos cartesianos, esquemas...). Recordemos que las representaciones semióticas pueden ser producciones discursivas o no discursivas. (Fig. 3).

Por ejemplo, en la pregunta 1 del $\mathrm{C}_{1}$, además del contexto conceptual, se genera un contexto geométrico dado por el lenguaje geométrico usado en el enunciado. Vinculado a este lenguaje está la coordinación de diferentes registros de representación semiótica. La primera parte del enunciado, el proceso de división infinita por mitades, está descrito con un registro semiótico lingüístico (lengua natural), dando paso posteriormente a un registro de representación semiótica no lingüístico, que es una figura geométrica (segmento de recta), terminando el enunciado en lengua natural.

El estudiante, entonces, no sólo tiene que resolver un mismo problema representado (en el sentido semiótico) de diferente forma, sino que ha de resolver un mismo problema expresado en diferentes lenguajes matemáticos. En este caso, decir que está representado de diferente forma, quiere decir que tiene representación distinta y lenguaje matemático distinto, lo que contextualiza ma- 
Figura 3

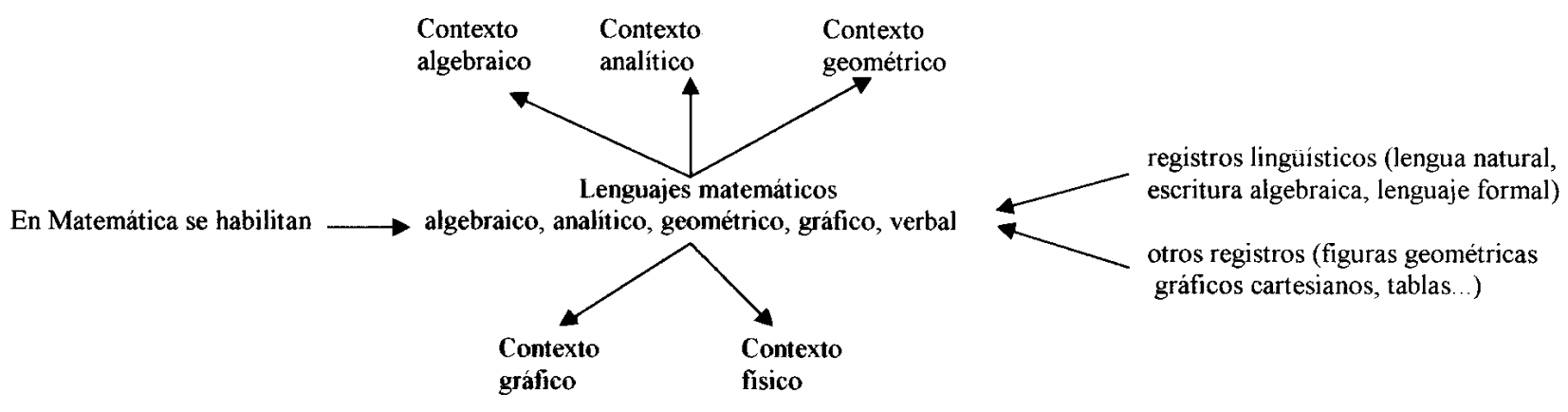

temáticamente el problema (por ejemplo, muchas veces el estudiante se enfrenta a esta situación en una guía de problemas o en un libro, al final de un tema, en una sección dedicada al aprendizaje de un concepto específico). Ante esta situación en que el estudiante tiene que resolver un mismo problema pero expresado de distintas maneras, se generan respuestas contradictorias entre sí (la de un problema con respecto a otro). Nosotros llamamos a estas respuestas contradictorias entre sí, respuestas incoherentes, o coherentes en caso contrario. Las líneas de coherencia son las que permiten identificar este tipo de respuestas coherentes o incoherentes. En consecuencia, podemos tener un alumno cuyas ideas o respuestas sean inconsistentes con el concepto involucrado (idea o respuesta errónea) y, sin embargo, mostrarse coherente en su pensamiento (ideas o respuestas equivalentes en problemas diferentes).

Las líneas de coherencia nos permiten determinar y definir lo que para nosotros es un alumno coherente con su propio pensamiento.

Un estudiante es considerado coherente, en nuestro estudio, si todas sus respuestas están en la misma línea de coherencia (1, 2 o 3). De esto se puede deducir que un alumno puede ser coherente con su pensamiento aunque sea inconsistente con el concepto matemático involucrado.

Por ejemplo, el alumno 26 (ver categoría 1, fig. 10) es coherente en la línea 1, sin embargo es un alumno finitista o que evade la infinitud.

Podemos hablar de tres tipos de alumnos:

1) Alumno coherente y consistente: tiene todas sus respuestas en la línea 2 .

2) Alumno coherente pero inconsistente: tiene todas sus respuestas en la línea 3 o en la línea 1.

3) Alumno incoherente: tiene respuestas que no son coherentes en alguna línea ( 1, 2 o 3); por ejemplo, podría tener tres respuestas en la línea 1 , dos respuestas en la línea 2 y una en la línea 3.
Teniendo en cuenta estos tres tipos de estudiantes y observando las figuras 10 a $14 \mathrm{del}$ anexo, correspondientes a las categorías de los estudiantes según las líneas de coherencia, vemos que el alumno 26 es coherente en la línea 1 y el 46 en la línea 2 . Curiosamente no hay ningún alumno de la categoría 3 que sea coherente, aunque cinco de ellos $(3,32,38,43,77)$ tienen una sola respuesta que no es coherente con la línea. Por ejemplo, en el caso del alumno 32, todas sus respuestas menos la primera corresponden a la línea 3. En la primera pregunta contesta que el punto de bisección no alcanza al punto B, ya que éste es punto extremo del segmento. Este alumno, si supera el obstáculo cognitivo específico, podría llegar a ser coherente en la línea 3.

En cada categoría construida hay de tres a seis respuestas coherentes; por tanto, el nivel o el grado de incoherencia de cada alumno es diferente. Es decir, no todo alumno incoherente lo es de la misma manera ni con la misma profundidad. Vinner catalogó dos niveles de inconsistencias: superficiales y profundas. Por el tipo de inconsistencias presentes en las respuestas de los alumnos que se han evidenciado en el estudio, pensamos que la mayoría son profundas. Creemos que esta profundidad también tiene distintos niveles. En algún alumno será más profunda que en otro.

Nos situamos ahora en las categorías 1, 2, 3, 1/3 y mixta: en cada categoría, de manera diferente a la que corresponde a la línea 2 , hay un número bajo de respuestas actualistas dadas por los estudiantes (Fig. 10-14).

En dos de ellas, la 3 y la 1/3 (Fig. 12-13), se puede decir que hay ausencia de respuestas actualistas2, lo cual llama la atención. En estos alumnos, el contexto y los registros de representación semiótica influyen en dos sentidos, hacia lo finito o evasión de lo infinito, y hacia una intuición potencial del infinito. Se podría decir que la noción potencial del infinito se mantiene profundamente arraigada en la mente del estudiante, ya que la influencia de representaciones acotadas no le llevan hacia una respuesta del tipo actual. Hay una mayor presencia de respuestas coherentes en el sentido potencial, que evidencian un mayor arraigo de la concepción 
potencial del infinito y una ausencia, aunque sea débil, de la noción actual del concepto.

En la categoría 1, a diferencia de la 3 y $1 / 3$, hay presencia de respuestas actualistas, aunque sean escasas. La presencia de este tipo de respuestas en el recorrido de algunos alumnos nos indica que la representación de cada problema influye de manera distinta en estos estudiantes que en los alumnos de las dos categorías anteriores. Cuando en la mente del sujeto lo infinito irrumpe en lo finito, es la mirada a los espacios acotados o el hacer una analogía a conceptos finitos lo que permite que acepte una primera presencia de un infinito actual.

Los estudiantes que pertenecen a la categoría mixta (Fig. 14) son muy sensibles a la representación de cada problema, como lo demuestra la poca coherencia que se observa en sus respuestas. Por otra parte, hay que distinguir entre los estudiantes que están situados en esta categoría sin ninguna respuesta en la línea 2, y los que sí la tienen.

Curiosamente, la categoría mixta es la que ha obtenido un mayor número de respuestas actualistas por parte de los estudiantes (sin considerar la línea 2). Esto indica que, en este grupo de estudiantes, la noción de infinito actual o la aceptación del infinito actual está presente en mayor grado que en los grupos anteriores. Parece que la noción potencial no está tan arraigada como en el grupo de la categoría 3 y que, tal vez, hay una mayor capacidad intuitiva para acoger la existencia de un infinito actual.
Lo expuesto anteriormente, mirando los tipos y origen de incoherencias de cada categoría, permite pensar y decir que las inconsistencias presentes en las respuestas de los estudiantes, derivadas principalmente de una intuición natural del infinito, tienen diferentes grados de profundidad en los alumnos.

\section{La tarea de conexión}

Teniendo como base la teoría cognitiva de las representaciones semióticas desarrollada por Duval y citada en el apartado dedicado al marco teórico, hemos descrito en nuestra investigación lo que entendemos por tarea de conexión y hemos reflexionado sobre su posible rol para lograr un pensamiento más coherente en el estudiante.

Supongamos que tenemos un problema matemático expresado de dos maneras diferentes (puede ser cualquier par de problemas de nuestro cuestionario $\mathrm{C}_{1}$ ). Tenemos los problemas A y B. Cada problema se expresa en un lenguaje matemático, lo que genera un contexto propio al mismo (Fig. 4). En cada uno se movilizan registros de representación semiótica que podrían resultar iguales o diferentes.

Se accede al objeto matemático a través de su representación. En el caso de nuestro estudio, debería ser el de infinito actual, aunque, como hemos visto, algunos de los registros de representaciones semióticas han inducido a los estudiantes a representarse como objeto a un infinito potencial. Por este hecho, en el gráfico (Fig. 4), la figura que indica la representación del objeto matemá-

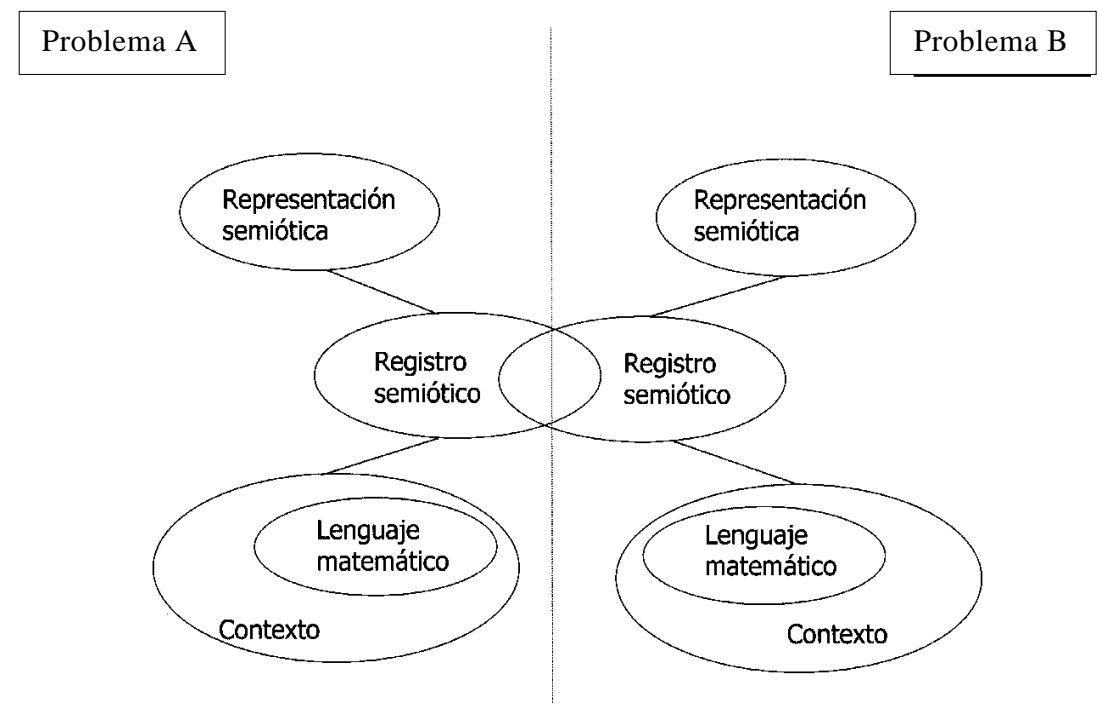


tico vinculado al problema A aparece de manera disjunta al del problema B, aunque en ambos la noción matemática sea la misma.

La situación teórica no cambiaría si los dos problemas utilizasen el mismo lenguaje matemático.

Aunque los elementos estructurales (lenguaje, sistemas semióticos, símbolos) son diferentes en los dos problemas (no necesariamente tendrían por qué serlo), el concepto matemático involucrado es el mismo. Creemos que es importante, como parte de la actividad matemática, saber identificar esta situación y saber utilizarla, ya que podría permitir no sólo obtener respuestas asociadas coherentes sino también disminuir la compartimentación del conocimiento.

No hemos mencionado todavía los resultados de $\mathrm{C}_{2}$, parte (b), donde algunos estudiantes afirmaron que las cinco preguntas o respuestas del $\mathrm{C}_{1}$ están relacionadas con el tema del infinito (27 alumnos); otros, que están relacionadas con la divisibilidad en mitades (17 alumnos); y, finalmente, 4 afirmaron que están relacionadas con el infinito y la divisibilidad en «mitades». Sin embargo, esto no ha sido suficiente para obtener respuestas asociadas coherentes; es necesario, además, saber utilizar y aprovechar la comprensión y el proceso de resolución utilizado en los diferentes problemas.

De manera análoga, hemos querido entender las conexiones, pero agregando una tarea que se realiza en la actividad matemática durante el proceso de aprendizaje de un concepto matemático, la tarea de conexión. En este caso la conexión se establece entre las representaciones; en el caso que estamos tratando, entre las representaciones correspondientes a cada problema (Fig. 5). La tarea de conexión consistiría en identificar y establecer relaciones entre los problemas, en cuanto a lenguaje matemático y registro de representación semiótica se refiere, y reconocer los contextos (conceptual y global) de los problemas, de manera que permita una influencia mutua, dando lugar a respuestas asociadas coherentes con los problemas.

Podemos ilustrar lo anterior con el siguiente ejemplo. Pensemos en los problemas 1 y 3 del $C_{1}$. La tarea de conexión entre los dos problemas consiste en reconocer que en ambas preguntas está presente la divisibilidad infinita por mitades con dos iteracciones y con naturaleza de contenido (contexto conceptual); son dos problemas que representan el mismo concepto, pero tales que, en el primero, se utiliza el lenguaje geométrico y, en el tercero, el analítico. Vinculados a los lenguajes matemáticos, usados en el enunciado de ambos problemas, estarían los registros de representación semiótica: figura geométrica (segmento, en la pregunta 1) y escritura numérica (suma infinita, en la pregunta 3); específicamente, en este punto, la tarea de conexión consistiría en reconocer los siguientes aspectos:

- Si se considera el segmento de dimensión 1, es decir, el segmento real $[0,1]$, cada punto del proceso de bisección se debe identificar con cada uno de los sumandos de la suma infinita de la pregunta 3 .

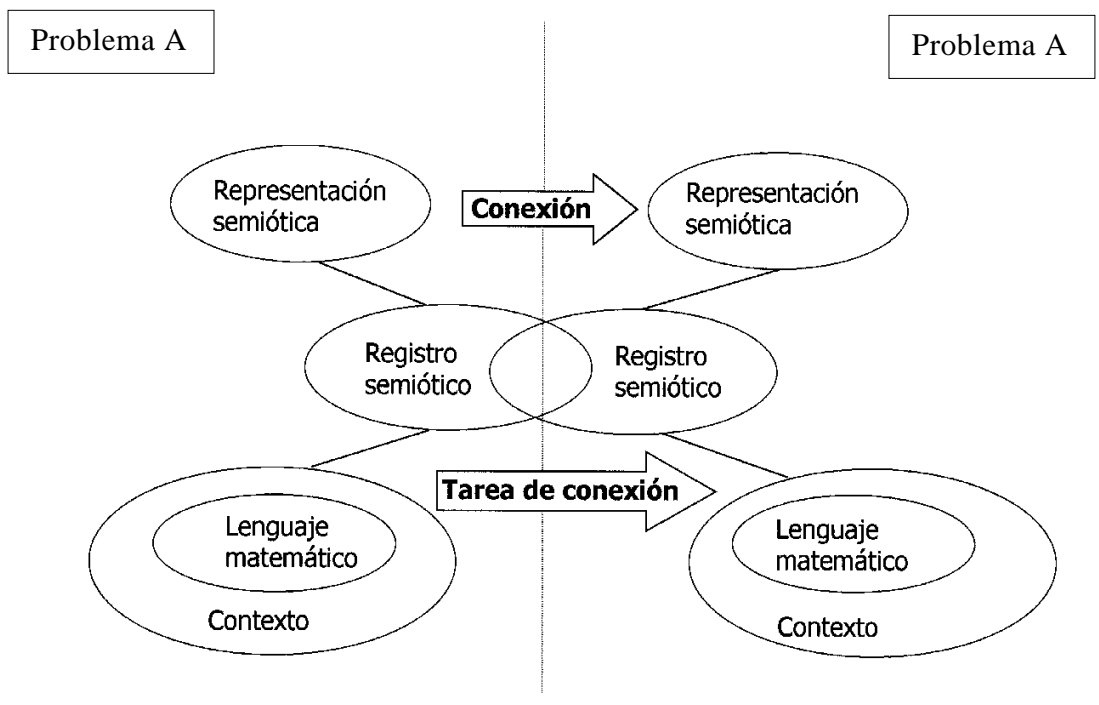


- La suma infinita del enunciado representa numéricamente la suma infinita de los segmentos que son resultado de las bisecciones y, por tanto, una solución explícita de la serie es la respuesta correcta a la primera pregunta.

- Una respuesta de la pregunta 1 debe ser asociada y coherente con la respuesta de la pregunta 3 .

Hablamos de coherencia y no de consistencia, dado que un alumno puede mantener una concepción de infinito potencial en una de las preguntas 1 o $3 \mathrm{y}$, al conectar las preguntas de la manera antes descrita, puede mantener tal concepción; es decir, mantiene la coherencia respecto a la noción matemática presente, pero no la consistencia.

También puede suceder que, en una de las preguntas, el sujeto tienda a una concepción potencial del infinito y, en la otra, a una concepción actual. En este caso, si hace la conexión entre las preguntas y es consciente de que ambas respuestas deben ser asociadas y coherentes, tendrá que optar entre la respuesta potencial y la actual, dejando en evidencia, en este caso, lo paradójico de los problemas. Es probable que, en esta situación, el alumno se deje llevar por la intuición natural.

Queremos subrayar que pensamos que esta tarea no es puntual sino que es un proceso no inmediato que aboca en la adquisición de una habilidad matemática.

\section{Inducción de la tarea de conexión durante las entrevis- tas}

Un ejemplo de lo que hemos afirmado en el párrafo anterior lo podemos dar a partir de nuestras entrevistas. Éstas se pensaron como instrumento de ayuda necesario para obtener información sobre la posible influencia de esta tarea de conexión en la búsqueda de coherencia.

En la figura 16 del anexo podemos ver el guión usado por la entrevistadora. Se puede observar que es una entrevista dirigida, en el sentido de que optamos por preguntas que permiten inducir al estudiante a establecer cierto tipo de conexiones entre las preguntas.

Se entrevistaron 6 estudiantes según la categorización realizada por líneas de coherencia, desestimando aquellos alumnos ubicados en las líneas 3 y 1/3. Estos alumnos básicamente tienen una concepción potencial del infinito, que es la natural, y se pensó que se obtendrían datos menos ricos que con aquellos alumnos que se encuentran en el resto de categorías y que se habían mostrado más influenciados por las representaciones de las preguntas. Los criterios usados para la selección han sido:

a) tener por lo menos una respuesta en la línea 2;

b) tener por lo menos un cambio de respuesta;

c) los comentarios escritos en los cuestionarios;

d) entre los alumnos que cumplen los requisitos y están en una misma categoría, seleccionar los que tengan respuestas diferentes a la primera pregunta.
Las preguntas planteadas fueron la 1,3 y 4 , en un primer tiempo de la entrevista, y la 2 , en un segundo tiempo, después que los estudiantes habían resuelto las tres primeras.

En la siguiente tabla podemos observar la situación de los estudiantes al final de la entrevista, con relación a la concepción del concepto y situación de coherenciaconsistencia de cada alumno.

\begin{tabular}{|l|l|l|l|}
\hline $\begin{array}{l}\text { Alumno } \\
\text { núm. }\end{array}$ & Concepción & $\begin{array}{l}\text { Coherencia } \\
\text { Incoherencia }\end{array}$ & $\begin{array}{l}\text { Consistencia } \\
\text { Inconsistencia }\end{array}$ \\
\hline 2 & $\begin{array}{l}\text { Conflicto- } \\
\text { paradoja }\end{array}$ & Coherente & Inconsistente \\
\hline 25 & $\begin{array}{l}\text { Actual en la } \\
\text { 1a. y 4a. pregunta. } \\
\text { Finitista en la } \\
\text { 3a. y 2a. pregunta }\end{array}$ & Incoherente & $\begin{array}{l}\text { Consistente en la } \\
\text { a. y 4a. pregunta. } \\
\text { Inconsistente en la } \\
\text { 3a. y 2a. pregunta. }\end{array}$ \\
\hline 42 & Potencial & Coherente & Inconsistente \\
\hline 47 & Actual & Coherente & Consistente \\
\hline 58 & Potencial & Coherente & Inconsistente \\
\hline 76 & Potencial & Coherente & Inconsistente \\
\hline
\end{tabular}

El análisis descriptivo e interpretativo de las entrevistas ha dejado en evidencia que, para que los alumnos lleguen a la situación final de coherencia, ha sido fundamental:

a) Reconocer en todas las preguntas el proceso de divisibilidad infinita, con los dos tipos de iteracciones, la divergente y la convergente.

b) Establecer la relación y la conexión entre las preguntas a través de la sucesión numérica.

Hemos identificado que dicha tarea puede además favorecer:

1) La aparición del conflicto y la consciencia de la paradoja en el estudiante cuando hay, por lo menos, una respuesta del tipo actualista, en algunos de los problemas, y cuando no hay presencia de conflicto o consciencia de la paradoja en la mente del alumno, previa a la tarea.

El siguiente diálogo puede ilustrar, como ejemplo, el conflicto que se va generando en el estudiante a lo largo de la entrevista. Corresponde a la parte de resolución de la segunda pregunta del $\mathrm{C}_{1}$. El alumno ya había resuelto previamente los tres primeros problemas correspondientes a la entrevista. Se puede observar una autobúsqueda de coherencia en el estudiante. En el cuestionario, después de haber descrito el recorrido de la pelota, este estudiante había contestado que la distancia que recorre se puede calcular sabiendo el número de rebotes (línea 1). 
Entrevistadora: «Y hace un medio de lo anterior y te están preguntando: ¿si hace un medio de lo anterior, cuánto recorrido hace?»

\section{$[\ldots]$}

Alumno: «Claro, si cada vez hace un medio del anterior, en un principio yo diría que... que claro, podría hacer infinitos botes $\mathrm{y}$, por lo tanto, se acerca cada vez, la distancia es infinita. Pero si ponemos en función de, bueno... en esta suma... sería dos veces..., bueno... y lo relaciono con lo que he dicho antes: si la unidad es dos, lo máximo vendría siendo cuatro, eso ya no lo sé.»

[Va escribiendo la suma que representa el recorrido de la pelota y sumando.]

Entrevistadora: « $\mathrm{Y}$, entonces, podrías calcular la distancia total recorrida por la pelota?»

Alumno: «No sé, no la veo... es que es la misma suma que el número dos; entonces, antes he dicho que esto se acercaba a dos, si esto ya son dos metros, más dos metros... esto es seis... No sé, ahora me parece que ya no sé mucho qué digo.»

Entrevistadora: «¿Por qué te asusta decir que esto es seis?»

Alumno: «No sé porque estoy diciendo todo lo que..., que me pasa. Es que, bueno, el día que nos pasaste esto ya iba escribiendo, pero las cosas que me parecía. Bueno, no había llegado a pensar, a hacer tantas relaciones, supongo que tampoco no estuve tanto tiempo... y por esto ahora digo esto porque son especulaciones que yo hago, son cosas que yo pienso, pero que quizás no las escribiría después... por eso ahora que lo voy diciendo..., quizás lo que voy diciendo... ¿Qué mal!

2) La «autobúsqueda» de coherencia, de manera consciente o no, en las respuestas y afirmaciones relacionadas con las preguntas (a través de la tarea se llega a una mayor consciencia de la semejanza de la situación planteada en cada problema). De esta manera, la tarea de conexión podría ayudar a regular y completar los procesos que son necesarios para la comprensión de cada problema.

Podemos ver como ejemplo el siguiente diálogo. La entrevistadora hace la pregunta después de que ya se había inducido en el estudiante parte de la tarea de conexión entre las preguntas 1 y 3 del cuestionario y se había identificado que los términos de la suma infinita representan numéricamente los puntos de bisección del segmento de la primera pregunta en el caso particular $[0,1]$.

Entrevistadora: «¿Sigues pensando aquí [se refiere a la 1a. pregunta] sí se llega al punto B si se sigue biseccionando, haciendo las divisiones, que de forma numérica sería esta sucesión [señala los sumandos de la segunda pregunta] _, , 1/ $8, \ldots$ ? Y en la segunda (pregunta 3 del $\mathrm{C}_{1}$ ), ¿sigues pensando que nunca se llega al 2? ¿Sigues pensando que ésas son las respuestas?»

Alumno: «No, tienen que ser las mismas [se refiere a las respuestas], que si aquí es sí, aquí tiene que ser sí, bueno que llega a 2 y, si aquí sí no llega a 2 , aquí no llega al punto B. No sé, creo... porque, si es la misma sucesión de puntos... es lo mismo que lo representen así o con un gráfico.»
3) La identificación del obstáculo cognitivo, creencia u error conceptual que no permite una respuesta consistente a la cuestión. Esto permite la intervención didáctica o docente adecuada según el tipo y grado de profundidad de la inconsistencia.

Esta tarea permitió, en la mayoría de los casos, superar las limitaciones de los registros de representación y la influencia del contexto eliminando aquellas respuestas fundamentadas en la representación y dejando en evidencia los aspectos antes mencionados. Hubo un solo caso (25) en que la entrevistadora no consiguió inducir, en su totalidad, la tarea de conexión. Las posibilidades y consciencia de la limitación de algunos de los registros de representación no han sido explotados en su totalidad y el alumno se ha dejado llevar por la representación del problema. La situación se puede observar en el siguiente diálogo. En esta parte de la entrevista, el alumno ya había identificado que la sucesión de puntos dada por las bisecciones del segmento $(0,1)$ era la misma que la dada por los sumandos de la suma infinita:

\section{Alumno: «En ésta podrías llegar al punto B, al 1.»}

Entrevistadora: «Haciendo esta sucesión, piensas que podrías llegar al punto 1, en la primera pregunta y en la segunda, no lo ves, ¿aunque relaciones que la sucesión es la misma?»

Alumno: «Sí, yo veo... es que aquí, como lo tengo representado pienso que aquí el B, pues, que puedes ir haciendo bisecciones, pero llegarás al punto $\mathrm{B}$ aquí no, aquí sigue al infinito [se refiere a la suma].»

En dos de los estudiantes entrevistados $(2,42)$, se evidencia la creencia de que, dada la infinitud del proceso, el punto de bisección no alcanza al punto extremo B: la suma se aproxima a 2 , la función se acerca a la recta $y=2$ y la pelota recorre una distancia que se aproxima a $6 \mathrm{~m}$, pero se deja la posibilidad de que en el infinito las respuestas puedan ser exactas; es decir, que es del tipo actualista. A modo de ejemplo, el alumno 2, refiriéndose a la cuarta pregunta del $\mathrm{C}_{1}$, afirma:

Alumno: «...tampoco podríamos determinar el valor de la función cuando $x$ se hace muy grande, exactamente el punto, el valor de $x$ que la función sería 2 , sino que cuando a medida que $x$ vaya creciendo la $y$ se acercará más a 2.»

Entrevistadora: «¿No se podrá decir, entonces, que la función sea 2 ? ¿O solamente se acerca?»

Alumno: «En el infinito... pero claro, ¿qué es el infinito? Como decimos, en el infinito, infinito quiere decir que hay un valor más grande, entonces, claro, sí, si cogemos, sí, en el infinito sería 2 , pero...

Dos alumnos $(58,76)$ mostraron claramente una concepción potencial de infinito sin considerar la posibilidad de que fuera posible la convergencia del proceso hacia un punto exacto. Estos últimos presentaron un nivel de inconsistencia más profundo que los tres mencionados anteriormente y no dieron ninguna respuesta del tipo actualista durante la entrevista. El alumno 76, refiriéndose a la primera pregunta del $\mathrm{C}_{1}$, expresó: 
Entrevistadora: «Matemáticamente cuántas veces puedes hacer esas divisiones?»

Alumno: «!Huy!, infinitas, hasta que te aburras, claro. Lo que pasa que habrá un momento que, si lo haces a lápiz, pues el grosor del lápiz no esto, pero, si lo vas haciendo, matemáticamente siempre tienes un punto.»

Entrevistadora: «Y matemáticamente, si son infinitas veces, ¿no podrás llegar?, porque físicamente yo no puedo hacer infinitos pasos.

Alumno: «No... Bueno... Pero matemáticamente sí, ésa es la diferencia; o sea, matemáticamente puedes hacer unas cosas que luego en realidad no son así.»

Entrevistadora: «Pero no te permite llegar de todas maneras. Pregunto, ¿matemáticamente aunque sea las infinitas veces no te permiten llegar al punto?»

Alumno: «Nunca llegarás al punto porque siempre habrá un punto entre donde estás tú, entre el punto siempre habrá una mitad, entonces nunca llegarás.»

\section{A MODO DE CONCLUSIÓN}

En este artículo hemos querido mostrar la conveniencia e importancia de haber distinguido los términos inconsistencia e incoherencia en nuestra investigación. Entre otras cosas, los tipos y el origen de incoherencias que se observaron en las categorías de coherencia establecidas permitieron identificar un diferente grado en la profundidad de las inconsistencias directas o del grado de construcción o presencia del concepto de infinito actual en los estudiantes. Teniendo en cuenta la distinción mencionada, hemos podido clasificar tres tipos de alumnos: alumno coherente y consistente, alumno coherente pero inconsistente y alumno incoherente. Es decir, en problemas de divisibilidad infinita en que está presente la noción de infinito actual, los alumnos pueden mantener respuestas coherentes y consistentes, coherentes pero inconsistentes o, dependiendo de la representación del problema, pueden dar una respuesta consistente o no con el concepto, que puede ser coherente o no con otra representación del mismo problema.

Hemos descrito lo que entendemos por tarea de conexión en la actividad matemática y hemos dejado explícito lo que ha favorecido la inducción de esta tarea durante las entrevistas realizadas en la investigación. Subrayamos la importancia de esta tarea en la actividad matemática, como ayuda para desarrollar un pensamiento coherente en el estudiante, de manera particular cuando está presente la noción de infinito actual en los problemas implicados en la tarea.
El trabajo realizado por la entrevistadora durante las entrevistas y los resultados obtenidos a partir de ésta, sustentan nuestra afirmación de que, así como se hace necesario intervenir didácticamente en las tareas de cambio y coordinación de los registros de representación, es importante tener en cuenta didácticamente, la tarea de conexión. Un profesor, al resolver en una práctica varios problemas que son representados de diferente manera pero que presentan la misma noción matemática, tendrá que ayudar a establecer la conexiones pertinentes a sus estudiantes, de manera que no sean problemas aislados. Una manera podría ser la de comenzar con el reconocimiento de las diferencias de la representación: reconocer el tipo de lenguaje y contexto, tipo de registro de representación semiótica usado en cada uno de ellos, y establecer las conexiones entre los sistemas semióticos presentes y los registros. Podría ser una manera de incidir en el desarrollo de un pensamiento consistente y menos compartimentado. De igual manera, en las guías de estudio o en las secciones dedicadas a problemas al final de un apartado de un libro dedicado a un concepto matemático específico, sería necesario hacer explícitas y presentes algunas indicaciones o preguntas que ayuden a establecer las conexiones entre los problemas o ejercicios presentados.

Nos parece importante subrayar que fomentar la tarea de conexión en los estudiantes no debería ser una labor puntual, sino que debería ser una práctica constante propia de la actividad del docente durante el proceso de enseñanza-aprendizaje. Proponemos esta tarea como facilitadora y queda un camino abierto para nuevas investigaciones que puedan, desde el punto de vista de la enseñanza, corroborar lo antes expresado: proponer y llevar experiencias didácticas o intervenciones didácticas concretas en el aula o diseñar alguna unidad de aprendizaje o guía de trabajo, teniendo en cuenta el binomio «coherencia/ consistencia» y la «tarea de conexión» evaluando y analizando los resultados de dichas experiencias.

\section{NOTA \\ ${ }^{1}$ Este artículo resume una parte de la tesis doctoral «Infinito actual: inconsistencias e incoherencias de estudiantes de 16-17 años», realizada por Sabrina Garbin, bajo la dirección de Carmen Azcárate en el programa de doctorado del Departamento de Didáctica de las Matemáticas y de las Ciencias Experimentales de la Universidad Autónoma de Barcelona. El trabajo ha sido realizado en el marco del proyecto «Pensamiento matemático avanzado: procesos cognitivos de aprendizaje y fenómenos de enseñanza» (CICYT, BXX2000-0069). \\ ${ }^{2}$ Pensamos que las pocas que hay en uno de los dos grupos de la categoría 3 no son significativas dentro de estas categorías.}




\section{REFERENCIAS BIBLIOGRÁFICAS}

AZCÁRATE, C. (1990). «La velocidad: introducción al concepto de derivada». Tesis doctoral. Universitat Autònoma de Barcelona.

ALPER, J. y BRIDGER, M. (1997). Mathematics, models and Zeno's paradoxes. Synthese, 110(1), pp. 143-166.

BEHR, M. y HAREL, G. (1990). Students' errors, misconceptions and cognitive conflicts in application of procedures. Focus on Learning Problems in Mathematics, 12, pp. 75-84.

BLAZEK, J. y SITIA, C. (1997). Riabilitazione del metodo di calcolo con grandezze infinitamente piccole. L'insegnamento della Matematica, 20A-B, 5, pp. 551-574.

BLISS, J. y OGBORN, J. (1979). The analysis of qualitative data. European Journal of Science Education, 1(4), pp. 427-440.

BLISS, J., MONK, M. y OGBORN, J. (1983). Qualitative Data Analysis for Educational Research. Londres: Crom Helm.

BOYER, C. (1992). Historia de la Matemática. Madrid: Alianza Editorial.

D’AMORE, B. (1996). L'infinito: storia di conflitti, di sorprese, di dubbi. La Matemática e la sua Didattica, 3, pp. 322-335.

D’AMORE, B. (1997). L'infinito in didattica della matemática. La Matemática e la sua Didattica, 3, pp. 289-305.

DUVAL, R. (1993). Registres de représentation sémiotique et fonctionnement cognitif. Annales de Didactique et de Sciences Cognitives, 5, pp. 37-65.

DUVAL, R. (1996). Quel cognitif retenir en didactique des mathématiques? Recherches en Didactique des Mathématiques, 6(3), pp. 349-382. (Versión consultada: Quale cognitivo per la didattica della matematica? La Matematica e la sua Didattica, 3, 1996, pp. 250-269).

DUVAL, R. (1999a). L'Apprendimento in matematica richiede un funzionamiento cognitivo specifico? La Matematica e la sua Didattica, 1, pp. 17-42.

DUVAL, R. (1999b). Representation, vision and visualization: cognitive functions in mathematical thinking, basic issues for learning. Actas del PME 23, pp. 3-26.

FISHBEIN, E. (1982). Intuition and proof. For the Learning of Mathematics, (3)2, pp. 9-19.

FISHBEIN, E. (1998). Conoscenza intuitiva e conoscenza logica nell'attività matematica. La Matematica e la sua Didattica, 4, pp. 365-401.

FISHBEIN, E., TIROSH, D. y HESS, P. (1979). The intuition of infinity. Educational Studies in Mathematics, 10, pp. 2-40.

GARBIN, S. (1998). «Esquemas conceptuales e incoherencias de estudiantes de bachillerato en relación con el concepto de infinito actual contextualizado en problemas expresados en diferentes lenguajes matemáticos: verbal, geométrico, gráfico, algebraico y numérico. Estudio exploratorio». Tesis de maestría. Universitat Autònoma de Barcelona.

GARBIN, S. (2000). «Infinito actual: inconsistencias e incoherencias de estudiantes de 16-17 años». Tesis doctoral. Universitat Autònoma de Barcelona.
GARBIN, S. y AZCÁRATE, C. (2000). Esquemas conceptuales e incoherencias con relación al concepto de infinito actual. Educación Matemática, 12(3), pp. 5-18.

GRAY, E. y TALL, D. (1994). Duality, ambiguity and flexibility: a «proceptual» view of simple arithmetic. Journal for Research in Mathematics Education, 25(2), pp. 116-140.

HARRISON, C. (1996). The three arrows of Zeno. Synthese, 107(2), pp. 271-293.

JANVIER, C. (1987). Translation processes in mathematics education, en Janvier, C. (ed.). Problems of Representation in the Teaching and Learning of Mathematics, pp. 27-31. Hillsdale, New Jersey, Londres: Lawrence Erlbaum Associates Publishers.

LATORRE, A. et al., (1996). Bases metodológicas de la investigación educativa. Barcelona: Editorial GR92.

MORENO, L.E. y WALDEGG, G. (1991). The conceptual evolution of actual mathematical infinity. Educational Studies in Mathematics, 22(3), pp. 211-231.

MURA, R. y MAURICE, L. (1997). L'infini, un ensemble de nombres? Enquête auprès de futurs enseignants et enseignantes. For the Learning of Mathematics, 17(3), pp. 28-35.

NÚÑEZ, E. (1994). Subdivision and small infinities: Zeno, paradoxes and cognition. Actas del PME 18, 3, pp. 368-375.

RISCOS, A. (1996). Aritmética infinita. Epsilon, 34, pp. 91-98.

ROMERO, C. (1996). Una investigación sobre los esquemas conceptuales del continuo. Ensayo de un cuestionario. Enseñanza de las Ciencias, 14(1), pp. 3-14.

RUCKER, R. (1995). Infinity and the Mind. Princeton University Press.

SIERSPINSKA, A. (1987). Humanities students and epistemological obstacles related to limits. Educational Studies in Mathematics, 18 , pp. 371-397.

STAVY, R. y TIROSH, D. (1996). The role of intuitive rules in science and mathematics education. European Journal of Teacher Education, 19(2), pp. 109-119.

STEFFE, L.P. (1990). Inconsistencies and Cognitive Conflict: a Constructivist's View. Focus on Learning Problems in Mathematics, 12(3-4). Summer \& Fall Editions.

TALL, D. (1980). The notion of infinite measuring numbers and its relevance to the intuition of infinity. Educational Studies in Mathematics, 11, pp. 271-284.

TALL, D. (1992). The transition to advanced mathematical thinking: functions, limits, infinity, and proof. Handbook of Research on Mathematics Teaching and Learning, pp. 495-511.

TALL, D. (1995). Cognitive growth in elementary and advanced mathematical thinking. Actas del PME 19, 1, pp. 61-75.

TALL, D. y VINNER, S. (1981). Concept image and concept definition in mathematics with particular references to limits and continuity. Educational Studies in Mathematics, 12(2), pp. 151-169. 
TIROSH, D. (1990). Inconsistencies in students' mathematical constructs. Focus on Learning Problems in Mathematics, 12, pp. 111-129.

TIROSH, D. (1991). The role of students' intuitions of infinity in teaching the cantorian theory en Tall, D. (ed.). Advanced Mathematical Thinking, pp.199-214. Dordrecht/Boston/ Londres: Kluwer Academic Publisher.

TIROSH, D. y GRAEBER, A. (1990). Inconsistency in preservice elementary teachers' beliefs about multiplication and division. Focus on Learning Problems in Mathematics, 12, pp. 65-74.

TSAMIR, P. y TIROSH, D. (1994). Comparing infinite sets: intuitions and representations. Actas del PME 18, 4, pp. 345-352.
TSAMIR, P. y TIROSH, D.(1995). Metacognizione e coerenza: il caso dell'infinito. La matemática e la sua didattica, 2, pp. $122-131$.

VINNER, S. (1990). Inconsistencies: their causes and function in learning mathematics. Focus on Learning Problems in Mathematics, 12 , pp. 85-97

VINNER, S. (1997). The pseudo-conceptual and the pseudoanalytical thought processes in mathematics learning. Educational Studies in Mathematics, 34(2), pp. 97-129.

WALDEGG, G. (1987). «Esquemas de respuesta ante el infinito matematico». Tesis doctoral. Mexico, DF.

WILSON, P. S. (1990) Inconsistent Ideas Related to Definitions and Examples. Focus in Learning Problems in Mathematics, 12(3-4), pp. 31-47.

[Artículo recibido en noviembre de 2000 y aceptado en diciembre de 2001.] 


\section{ANEXO}

Figura 6

Primera línea de coherencia.

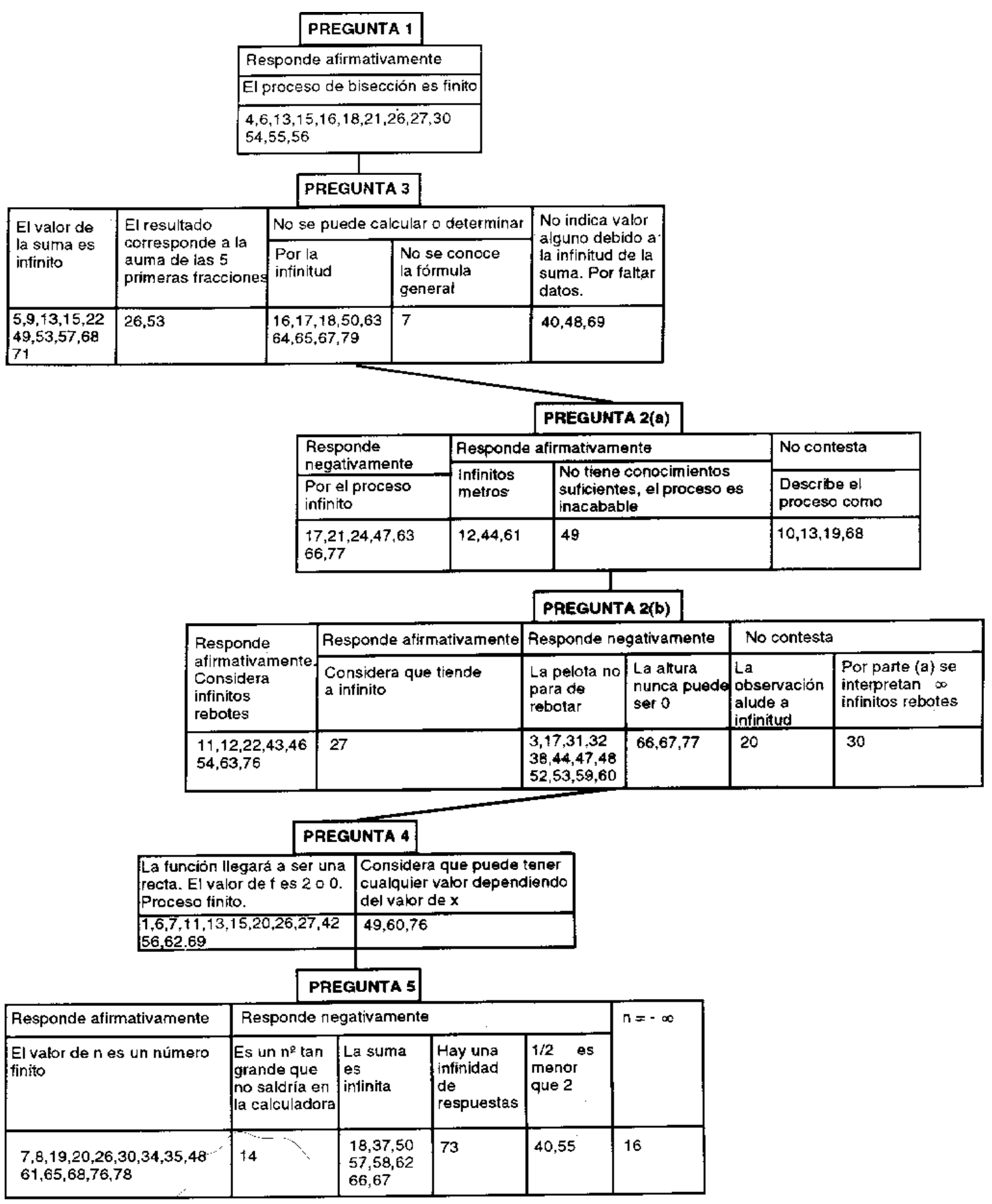


Figura 7

Primera línea de coherencia. Línea 1.1.

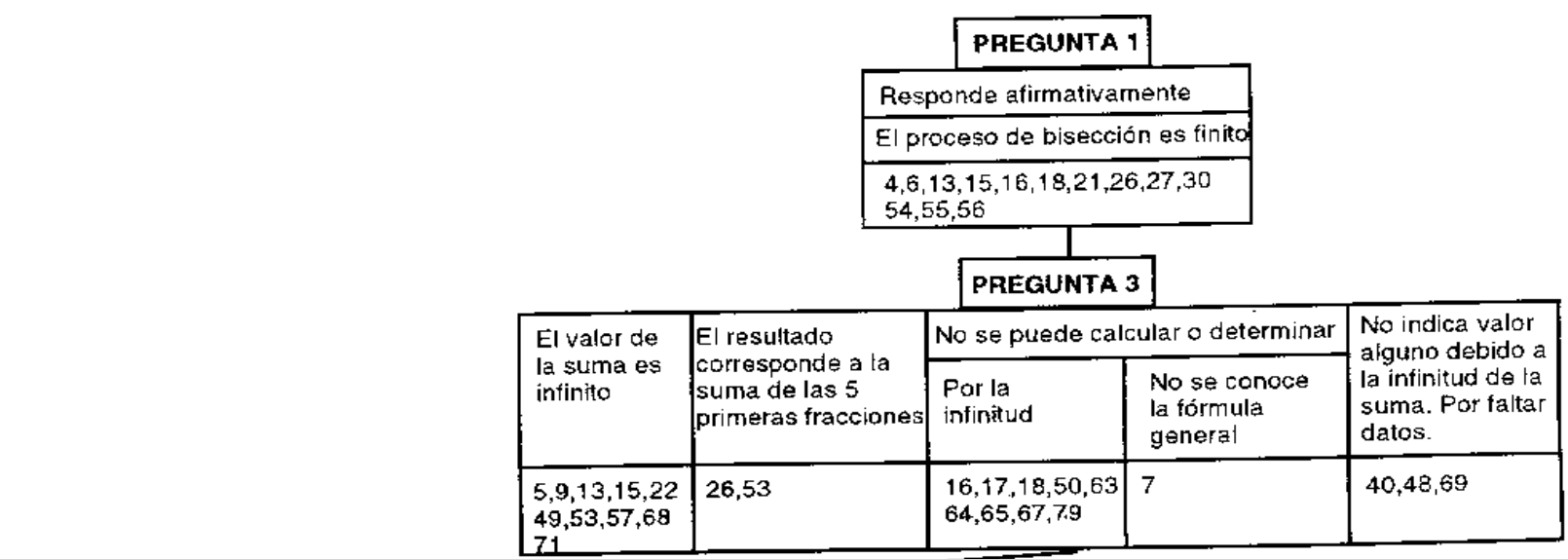

\begin{tabular}{|c|c|c|c|c|c|c|c|c|c|c|}
\hline \multirow{3}{*}{$\begin{array}{l}\text { Responde } \\
\text { afirmativamente }\end{array}$} & & & & & PREGUN & TA 2(a) & & & & \\
\hline & \multirow{2}{*}{\multicolumn{2}{|c|}{$\begin{array}{l}\text { Se considera } \\
\text { la necesidad } \\
\text { de conocer el } \\
n^{2} \text { de rebotes } \\
\text { tanto para } \\
\text { atimar como } \\
\text { para negar }\end{array}$}} & \multirow{2}{*}{\multicolumn{2}{|c|}{$\begin{array}{l}\text { Se considera } \\
\text { que no hay } \\
\text { datos } \\
\text { suficientes } \\
\text { para afirmar } \\
\text { o negar }\end{array}$}} & \multicolumn{2}{|c|}{$\begin{array}{l}\text { Responde } \\
\text { afirmativamente }\end{array}$} & \multicolumn{2}{|c|}{$\begin{array}{l}\text { Responde } \\
\text { negativamente }\end{array}$} & \multicolumn{2}{|l|}{ No contesta } \\
\hline & & & & & \multicolumn{2}{|c|}{$\begin{array}{l}\text { Se considera el } \\
\text { comportamiento } \\
\text { de la pelota. } \\
\text { Alude a finitud. }\end{array}$} & \multicolumn{2}{|c|}{$\begin{array}{l}\text { La pelota tiene } \\
\text { solo un } \\
\text { movimiento } \\
\text { vertical }\end{array}$} & $\begin{array}{l}\text { La distancia } \\
\text { depende de } \\
\text { lo que haga } \\
\text { la pelota }\end{array}$ & $\begin{array}{l}\text { Escribe } \\
\text { tormulas } \\
\text { físicas. No } \\
\text { tiene datos } \\
\text { suficientes }\end{array}$ \\
\hline \multirow[t]{2}{*}{$\begin{array}{l}11,28,33,34,35 \\
36,39,65,73,76\end{array}$} & \multicolumn{2}{|c|}{$\begin{array}{l}2,3,9,16,23 \\
31,38,56,59 \\
72,74\end{array}$} & \multicolumn{2}{|c|}{$\begin{array}{l}5,6,18,22,25 \\
27,41,45,50 \\
51,53,55,57 \\
64,69,70,71\end{array}$} & \multicolumn{2}{|c|}{$\begin{array}{l}15,40,42,58,62 \\
67,79,80\end{array}$} & \multicolumn{2}{|l|}{1} & 4 & 8,26 \\
\hline & & & & & \multicolumn{2}{|c|}{ PREGUATA 2(b) } & & & & \\
\hline \multicolumn{5}{|c|}{ Responde afirmativamente } & & \multicolumn{3}{|c|}{ Responde negativamente } & \multicolumn{2}{|c|}{ No contesta } \\
\hline \multirow{2}{*}{\multicolumn{2}{|c|}{$\begin{array}{l}\text { Considera } \\
\text { finitos } \\
\text { febotes }\end{array}$}} & \multicolumn{4}{|c|}{ No indica una cantidad } & \multirow{2}{*}{\multicolumn{2}{|c|}{$\begin{array}{l}\text { No hay } \\
\text { datos } \\
\text { suficientes }\end{array}$}} & \multirow{2}{*}{$\begin{array}{l}\text { Depende de } \\
\text { como se } \\
\text { tire la } \\
\text { pelota }\end{array}$} & \multirow{2}{*}{$\begin{array}{l}\text { Alude a } \\
\text { finitud }\end{array}$} & \multirow{2}{*}{$\begin{array}{l}\text { Escribe } \\
\text { fómulas } \\
\text { fisicas }\end{array}$} \\
\hline & & \multicolumn{2}{|c|}{$\begin{array}{l}\text { Conociendo } \\
\text { a gunos datos }\end{array}$} & \multicolumn{2}{|c|}{$\begin{array}{l}\text { Aluce a } \\
\text { finitud }\end{array}$} & & & & & \\
\hline \multicolumn{2}{|c|}{$\begin{array}{l}10,13,16,24,28,29,94 \\
35,36,39,42,56,61,65 \\
68,79\end{array}$} & \multicolumn{2}{|c|}{$\begin{array}{l}5,15,19,41,50,57 \\
58,62,64,71,74\end{array}$} & \multicolumn{2}{|c|}{$\begin{array}{l}1,4,6,7,9,14,21 \\
23,37,40,49,67\end{array}$} & \multicolumn{2}{|c|}{$\begin{array}{l}+8,25,26,51 \\
55,69,70,73\end{array}$} & 78 & $33,45,80$ & 8 \\
\hline
\end{tabular}

PRECUNTA 4

\begin{tabular}{|c|c|c|c|c|c|}
\hline \multicolumn{2}{|c|}{$\begin{array}{l}\text { La función llegará a ser una } \\
\text { recta. E | valor de } 1 \text { es } 200 . \\
\text { Proceso finito. }\end{array}$} & \multicolumn{3}{|c|}{$\begin{array}{l}\text { Considera que puede tener } \\
\text { etalquier valor dependiendo } \\
\text { del valor de } x\end{array}$} & \\
\hline \multicolumn{2}{|c|}{$\begin{array}{l}1,6,7,11,13,15,20,26,27,42 \\
56,62,69\end{array}$} & \multicolumn{3}{|c|}{$49,60,76$} & \\
\hline & \multicolumn{4}{|c|}{ PREGUNTA 5} & \\
\hline afirma:ivamente & \multicolumn{5}{|c|}{ Responde negativamente } \\
\hline de nes un número & \multicolumn{2}{|c|}{$\begin{array}{l}\text { Es un: } n^{2} \text { tan } \\
\text { grande que } \\
\text { no saldria en } \\
\text { la calouladora }\end{array}$} & $\begin{array}{l}\text { La suma } \\
\text { es } \\
\text { infinita }\end{array}$ & $\begin{array}{l}\text { Hay una } \\
\text { infinidad } \\
\text { de } \\
\text { respuestas }\end{array}$ & $\begin{array}{l}1 / 2 \text { es } \\
\text { menor } \\
\text { que } 2\end{array}$ \\
\hline $\begin{array}{l}26,30,34,35,48 \\
76,78\end{array}$ & \multicolumn{2}{|l|}{14} & $\begin{array}{l}18,37,50 \\
57,58,62 \\
66,67\end{array}$ & 73 & 40,55 \\
\hline
\end{tabular}


Figura 8

Segunda línea de coherencia.

\section{PREGUNTA 1}

\begin{tabular}{|c|}
\hline Responde afirmativamente \\
\hline El proceso de bisección es $x$ \\
\hline $1229,40,42,46,47,59$ \\
\hline PREGUNTA 3 \\
\hline
\end{tabular}

\begin{tabular}{|l|l|}
$\begin{array}{l}\text { El resultado de la } \\
\text { suma es 2 }\end{array}$ & $\begin{array}{l}\text { El resultado de ta suma } \\
\text { es 1, 0 o un no decimal }\end{array}$ \\
\hline $\begin{array}{l}10,12,24,29,30,46 \\
51,54,58,76\end{array}$ & $11,35,47,55$ \\
\hline \multicolumn{2}{|c|}{} \\
\hline \multicolumn{2}{|c|}{ PREGUNTA 2(a) } \\
\hline
\end{tabular}

\begin{tabular}{|l|}
\hline Responde atirmativamente \\
\hline $\begin{array}{l}\text { Indica una distancia. } \\
\text { El proceso es infinito }\end{array}$ \\
\hline $30,46,54$ \\
\hline
\end{tabular}

PREGUNTA 2(b)

\begin{tabular}{|c|c|c|c|}
\hline $\begin{array}{l}\text { Responde } \\
\text { afirmativamente }\end{array}$ & \multicolumn{2}{|c|}{ Fiesponde afirmativamente } & No contesta \\
\hline Infinitos reboles & \multicolumn{2}{|c|}{$\begin{array}{l}\text { No indica cantidad. } \\
\text { Tiende a intinito. }\end{array}$} & $\begin{array}{l}\text { Observación } \\
\text { alude a } \infty\end{array}$ \\
\hline $11,12,22,43,44,46,54,63,76$ & \multicolumn{2}{|l|}{27} & 20 \\
\hline & \multicolumn{2}{|c|}{ PREGUNTA 4} & \\
\hline & \multicolumn{3}{|c|}{$\begin{array}{l}\text { La función liegará a ser una recta. } \\
\text { El valor de } \mathrm{I} \text { es } 200 \text {. } \\
\text { El proceso es infinito. }\end{array}$} \\
\hline & \multicolumn{3}{|c|}{$2,12,18,21,24,28,39,44,46,47,54$} \\
\hline & \multicolumn{2}{|c|}{ PREGUNTA 5} & \\
\hline & $=\infty$ & \multicolumn{2}{|c|}{ Responde negativamente } \\
\hline & \multirow{2}{*}{$\begin{array}{l}2,12,22,24,25,29 \\
29,36,39,42,44,45 \\
46,54,59,69,70,75\end{array}$} & \multicolumn{2}{|c|}{$\begin{array}{l}\text { Será un número } \infty \text { que } \\
\text { no conacemos. }\end{array}$} \\
\hline & & \multicolumn{2}{|l|}{49} \\
\hline
\end{tabular}


Figura 9

Tercera línea de coherencia.

PREGUNTA 1

\begin{tabular}{|l|}
\hline Responde negativamente \\
\hline $\begin{array}{l}\text { Por la infinitud del proceso } \\
\text { se aproxima, se acerca a } \mathrm{B}\end{array}$ \\
\hline \begin{tabular}{l}
$3,8,9,10,17,20,22,24,33,34$ \\
$35,37,38,43,44,45,49,50,52$ \\
$53,58,61,62,63,66,67,68,69$ \\
$70,71,72,73,74,75,77,78$ \\
\hline
\end{tabular} \\
\hline
\end{tabular}

PREGUNTA 3

\begin{tabular}{|c|c|c|c|c|c|c|}
\hline \multirow{2}{*}{$\begin{array}{l}\text { El resultado } \\
\text { de la suma } \\
\text { es } 1,99 . . . \\
2,99 \ldots\end{array}$} & \multirow{2}{*}{$\begin{array}{l}\text { El máximo } \\
\text { valor que } \\
\text { puede llegar } \\
\text { es } 1.99 . .\end{array}$} & \multicolumn{3}{|c|}{ No se puede calcular } & \multirow{2}{*}{$\begin{array}{l}\text { El valor de la } \\
\text { suma está } \\
\text { entré } \\
2 \text { y } 2,99 . .\end{array}$} & \multirow{2}{*}{$\begin{array}{l}\text { El vaior de la } \\
\text { suma se } \\
\text { aproxima a } 2\end{array}$} \\
\hline & & $\begin{array}{l}\text { No se puede } \\
\text { calcular un } \\
n^{0} \text { exacto }\end{array}$ & $\begin{array}{l}\text { Los sumandos } \\
\text { cada vez se hacen } \\
\text { más pequeños }\end{array}$ & $\begin{array}{c}\text { El } n^{q} \text { está } \\
\text { alrededor } \\
\text { del } 2 \\
\end{array}$ & & \\
\hline $\begin{array}{l}4,8,23,41,42 \\
59\end{array}$ & 75 & 20 & 16,63 & 73 & 74 & $\begin{array}{l}1,2,3,6,14,19,21 \\
27,28,31,32,33 \\
34,36,37,38,39 \\
43,44,45,52,60 \\
61,62,66,72,77 \\
78,90\end{array}$ \\
\hline
\end{tabular}

PREGUNTA 2(a)

Responde afirmativamente

El proceso es infinito $y$ la

distancia se aproxima a

$6 \mathrm{~m}$ o es $5,99 \ldots$

$14,29,32,37,43,48$

PREGUNTA 2(b)

\begin{tabular}{|c|c|c|c|c|c|}
\hline \multicolumn{2}{|c|}{ Responde afirmativamente } & \multicolumn{2}{|c|}{ Responde negativamente } & \multicolumn{2}{|l|}{ No contesta } \\
\hline \multirow[t]{2}{*}{$\begin{array}{l}\text { Infinitos } \\
\text { rebotes }\end{array}$} & $\begin{array}{l}\text { No indica } \\
\text { cantidad }\end{array}$ & \multirow[t]{2}{*}{$\begin{array}{l}\text { La pelota nunca } \\
\text { para de rabotar }\end{array}$} & \multirow[t]{2}{*}{$\begin{array}{l}\text { La altura nunca } \\
\text { puede ser } 0\end{array}$} & \multirow{2}{*}{$\begin{array}{l}\text { Observacion } \\
\text { alude a } \\
\text { infinitud }\end{array}$} & \multirow{2}{*}{$\begin{array}{c}\text { Por parte (a) } \\
\text { se interpreta } \\
\text { o reboles }\end{array}$} \\
\hline & Tiende a or & & & & \\
\hline $\begin{array}{l}11,12,22,43 \\
46,54,63,76\end{array}$ & 27 & $\begin{array}{l}3,17,31,32,38,44 \\
47,48,52,53,59,60\end{array}$ & $66,67,77$ & 20 & 30 \\
\hline
\end{tabular}

PREGUNTA 4

\begin{tabular}{|l|l|}
\hline $\begin{array}{l}\text { La tunción se aproxima } \\
\text { a } z, \text { al eje } x \text { o } 0\end{array}$ & $\begin{array}{l}\text { No se puede determinar. } \\
\text { Por la infinitud del proceso. } \\
\text { Nunca sera 2. }\end{array}$ \\
\hline $\begin{array}{l}3,4,5,8,9,10,14,17,19,23,29 \\
31,32,33,36,37,38,41,43 \\
48,50,52,53,55,58,59,64,66\end{array}$ & 16,63 \\
$67,70,71,72,73,77$ & \\
\hline
\end{tabular}

PREGUNTA 5

\begin{tabular}{|c|c|}
\hline \multirow{2}{*}{$\begin{array}{l}n=\infty \text { pero la suma } \\
\text { no liegará a } 2\end{array}$} & Responde negativamente \\
\hline & La suma nunca será 2 \\
\hline $23,24,42,45,47,59,60$ & $\begin{array}{l}1,3,4,6,9,17,21,31,32,33 \\
38,41,43,53,64,71,77,80\end{array}$ \\
\hline
\end{tabular}




\section{Figura 10}
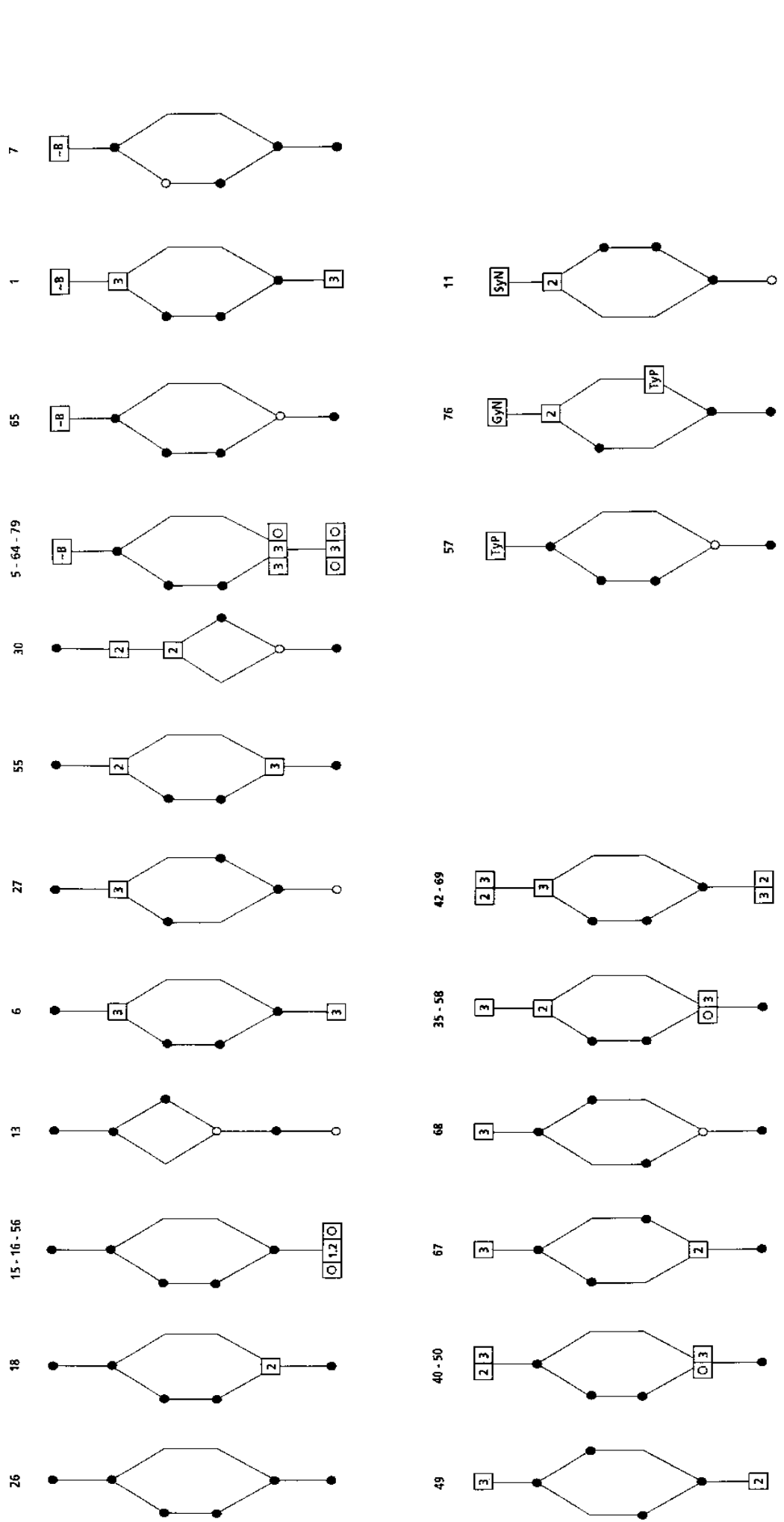

F

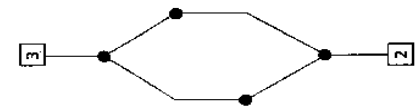

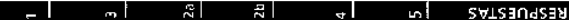



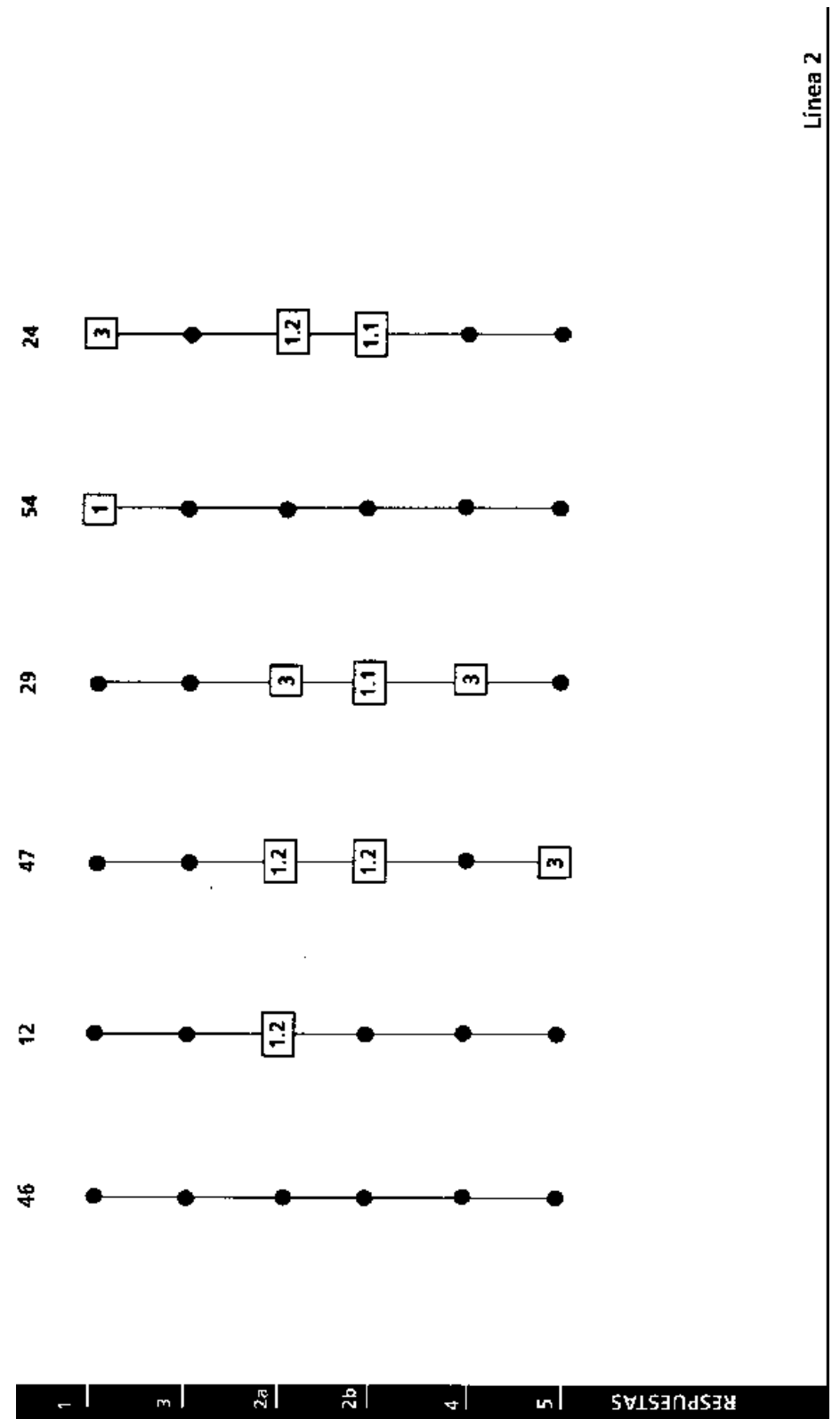

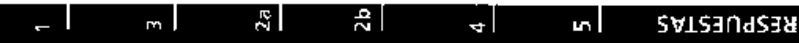


Figura 12

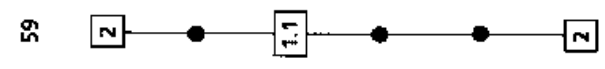

6

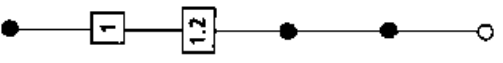

垨

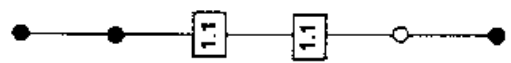

8
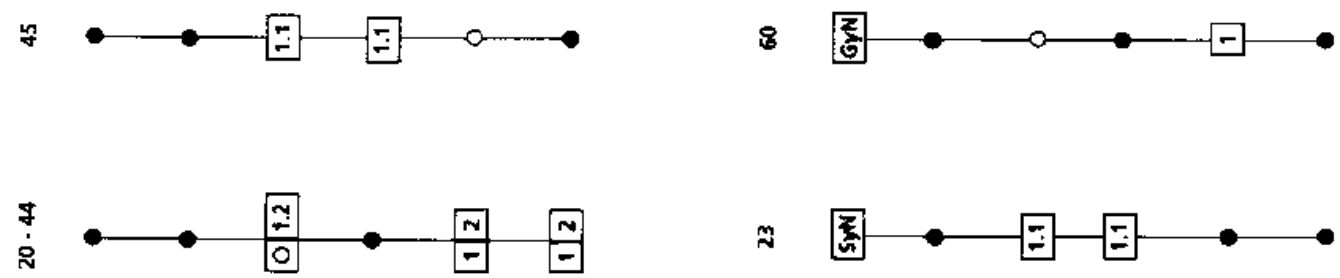

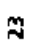
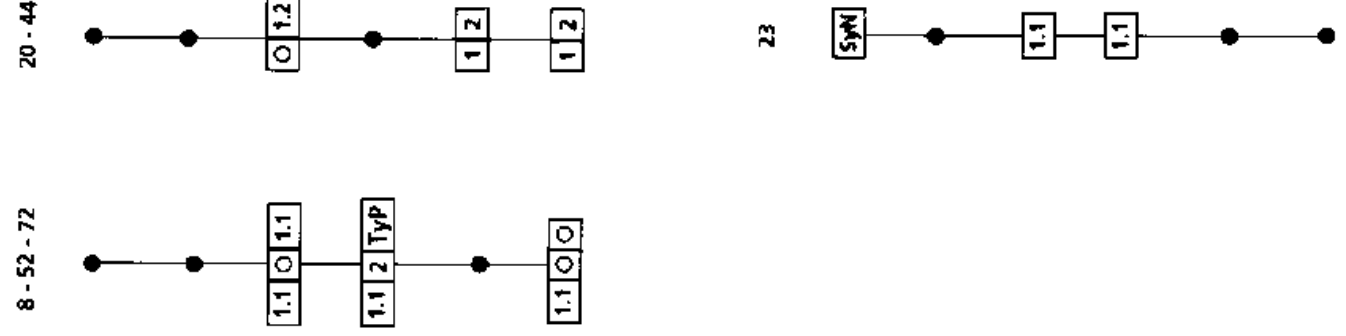

$\mathbf{m}$

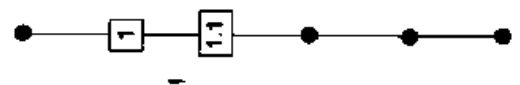

$\stackrel{m}{m} \bullet \quad \square \longrightarrow$

品
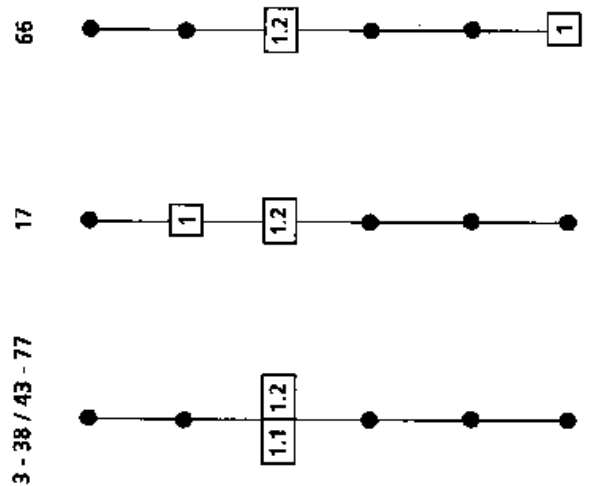

$\pm \quad \square \quad F$

$\bar{m} \longrightarrow$
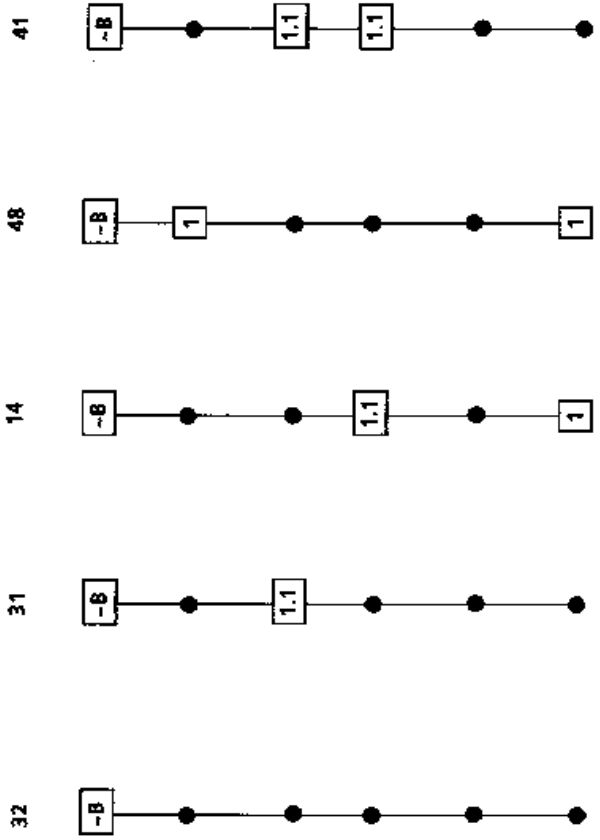
Figura 13

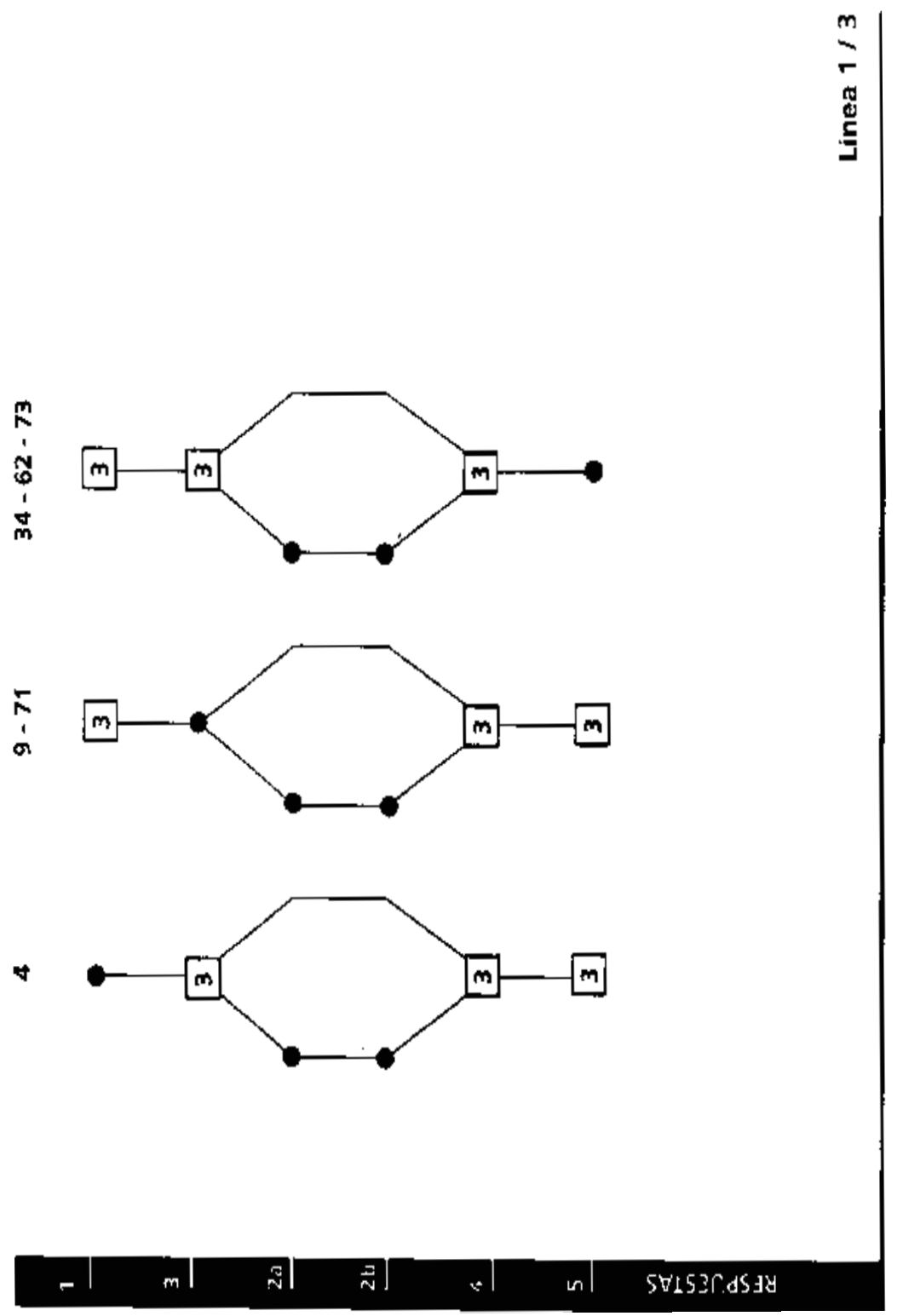


$\bar{N}-m-m$

$m$ m- $m-0$

$\circ m-m$

$\therefore m-F-m$

N $m-F-F$

$\%$ m $m-F$

$\bar{m}-m-a$

$F \quad m-m-O-O$ in $0-F$

$\Rightarrow \quad$ 密

$* \quad m-m-m$
$8 \quad m-F$

in

$\infty \quad m-m-m$ 


\begin{tabular}{|c|l|}
\hline Símbolo & \multicolumn{1}{|c|}{ Significado } \\
\hline 1 & La respuesta corresponde a la línea 1. \\
\hline 1.1 & La respuesta corresponde a la linea 1.1. \\
\hline 1.2 & La respuesta corresponde a la línea 1.2. \\
\hline 2 & La respuesta corresponde a la línea 2. \\
\hline 3 & La respuesta corresponde a la línea 3. \\
\hline$\sim \mathrm{B}$ & $\begin{array}{l}\text { En la pregunta l, el punto de bisección no puede } \\
\text { coincidir con B por ser este punto extremo del } \\
\text { segmento. }\end{array}$ \\
\hline T y P & $\begin{array}{l}\text { El alumno en su respuesta distingue entre la teoria } \\
\text { y la práctica. }\end{array}$ \\
\hline S y N & $\begin{array}{l}\text { El alumno responde: Si y No. Acepta como cierta } \\
\text { tanto la respuesta negativa como la afirmativa. }\end{array}$ \\
\hline G y N & $\begin{array}{l}\text { El alumno en su respuesta distingue entre la } \\
\text { representación geométrica y la numérica. }\end{array}$ \\
\hline$\bullet$ & $\begin{array}{l}\text { La respuesta corresponde a la línea de la categoría } \\
\text { en que está siturado el alumno. }\end{array}$ \\
\hline o & $\begin{array}{l}\text { El alumno no contesta a la pregunta. No entiende } \\
\text { la pregunta o sólo hace alguna observación. }\end{array}$ \\
\hline
\end{tabular}




\section{GUIÓN DE LA ENTREVISTA}

- Se pide al alumno que lea detenidamente los tres problemas.

- Se sugiere al alumno que resuelva los problemas en voz alta:

"ahora... pensemos en voz alta... cuando se piensa en un problema, son muchos los pensamientos, cuestiones, planteamientos, dudas, relaciones, etc., que nos surgen... no tengas miedo a expresarlos".

- Se introduce al alumno en la tarea de conexión.

¿Cómo conectarías ahora las 3 preguntas? ¿Qué elementos, conceptos, relaciones, propiedades, etc. tomarías en cuenta para conectar las preguntas?

- Podríamos comenzar con la primera...

trata de conectar la $1^{\mathrm{a}}$ con la $2^{\mathrm{a}}$

la $2^{\mathrm{a}}$ con la $3^{\mathrm{a}}$ y la $1^{\mathrm{a}}$ con la $3^{\mathrm{a}}$.

En caso de que sea necesario, por el tipo de respuesta del alumno, dirigir la tarea con las siguientes preguntas:

- Con el proceso descrito en la $1^{\text {a }}$ pregunta, ¿podrías construir una sucesión descendente de puntos?

(en caso que solo indique los puntos de bisección sin dale ningún valor numérico sugerir: imagínate que el segmento $A B$ es el segmento 01).

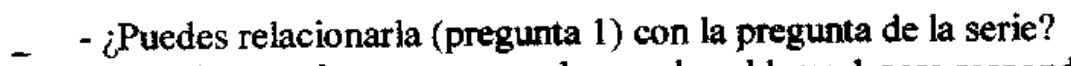
¿En qué te ayuda esta manera de ver al problema 1 para responder la segunda pregunta?

- ¿Qué puedes decir ahora de la tercera?.

- Se sitúa al estudiante en la pregunta 2 del cuestionario. Se le proporciona una hoja con el problema escrito para que lo lea. Si recordamos nuevamente el caso de la pelota, ipodrías usar como modelo algunas de estas preguntas para resolverlas?.

En caso de que sea necesario según la respuesta del estudiante:

- Piensa en el recorrido de la pelota y trata de representarlo.

- Se sitúa al estudiante ante una afirmación global.

- Considera la siguiente afirmación: "estos problemas son una representación geométrica, numérica y gráfica (respectivamente) de una misma situación matemática".

¿Estás de acuerdo con esta afirmación?

¿A qué situación se refiere? 